\title{
Static Plane-Symmetric Nonlinear Spinor and Scalar Fields in GR
}

\author{
Bijan Saha ${ }^{1,3}$ and G. N. Shikin ${ }^{2}$ \\ Received January 11, 2005; accepted March 30, 2005
}

\begin{abstract}
We consider a system of minimally coupled nonlinear spinor and scalar fields within the scope of a plane-symmetric gravitational field. The gravitational field plays crucial role in the formation of soliton-like solutions, i.e., solutions with limited total energy, spin, and charge. The change of the sign of the scalar field energy density of the system in question realizes physically if and only if the scalar charge does not exceed some critical value. In case of spinor field no such restriction on its parameter occurs. The choice of spinor field nonlinearity leads to the elimination of scalar field contribution to the metric functions, but leaves its contribution to the total energy unaltered. The spinor field is more sensitive to the gravitational field than the scalar field.
\end{abstract}

KEY WORDS: nonlinear spinor field (NLSF); nonlinear scalar field; plane-symmetric metric.

\section{INTRODUCTION}

In recent years, cosmological models exhibiting plane symmetry have attracted the attention of many specialists. At the present state of evolution, the universe is spherically symmetric and the matter distribution in it is isotropic and homogeneous. But at the early stage of evolution, it could have not had a smoothedout picture. Close to the big bang singularity, neither the assumption of spherical symmetry nor the isotropy can be strictly valid. That is why many authors consider plane symmetry, which is less restrictive than spherical symmetry and provides an avenue to study early days inhomogeneities. Note that, inhomogeneous cosmological models play an important role in understanding some essential features of

${ }^{1}$ Laboratory of Information Technologies, Joint Institute for Nuclear Research, Dubna, 141980 Dubna, Moscow, Russia.

${ }^{2}$ Department of Theoretical Physics, Peoples' Friendship University of Russia 6, Miklukho Maklay Street, 117198 Moscow, Russia.

${ }^{3}$ To whom correspondence should be addressed at Laboratory of Information Technologies, Joint Institute for Nuclear Research, Dubna 141980 Dubna, Moscow, Russia; e-mail: saha@thsun1.jinr.ru; URL: http://thsun1.jinr.ru/saha/ 
the universe such as the formation of galaxies during the early stages of evolution and the process of homogenization.

On the other hand, nonlinear phenomena have been one of the most popular topics during last years. Nevertheless, it must be admitted that nonlinear classical fields have not received general consideration. This is probably due to the mathematical difficulties which arise because of the nonrenormalizability of the Fermi and other nonlinear couplings (Ranada, 1983). In spite of this, the nonlinear generalization of classical field theory remains one of the possible ways to overcome the difficulties of a theory which considers elementary particles as mathematical points. In this approach, a hope to create a divergence-free particle theory was connected with a search for and studies of exact, regular, localized solutions to classical nonlinear field equations, able to describe the complicated spatial structure of particles observed in the experiment (Thirring and Skyrme, 1962). The gravitational field equation is nonlinear by nature and the field itself is universal and unscreenable. These properties lead to a definite physical interest in the gravitational field that goes with these matter fields. This project was realized in a number of papers where the authors thoroughly studied the scalar and/or electromagnetic field(s) in spherically and cylindrically space-time (Bronnikov and Shikin, 1991, 2001; Rybakov et al., 1992, 1994, 1997, 1998; Saha, 2000). Nevertheless, papers, dealing with soliton-like solutions of nonlinear field equations, ignore the proper gravitational field ${ }^{4}$ in the initial field system more often than not.

\subsection{Spinor Field Nonlinearity}

Nonlinear self-couplings of the spinor fields may arise as a consequence of the geometrical structure of the space-time and, more precisely, because of the existence of torsion. As early as 1938, Ivanenko (1938, 1947; Rodichev, 1961) showed that a relativistic theory imposes in some cases a fourth-order selfcoupling. In 1950, Weyl (1950) proved that, if the affine and the metric properties of the space-time are taken as independent, the spinor field obeys either a linear equation in a space with torsion or a nonlinear one in a Riemannian space. As the self-action is of spin-spin type, it allows the assignment of a dynamical role to the spin and offers a clue about the origin of the nonlinearities. This question was further clarified in some important papers by Utiyama (1956), Kibble (1960) and Sciama (1960). In the simplest scheme the self-action is of pseudo-vector type, but it can be shown that one can also get a scalar coupling (Ranada and Soler, 1972). An excellent review of the problem may be found in (Hehl et al., 1976).

\footnotetext{
${ }^{4}$ By proper gravitational field here we mean the following: the matter field acts as the source of the gravitational field. The gravitational field, on its part, effectively influences matter field configuration. In that sense, the "proper" gravitational field is the solution of the self-consistent system of matter and gravitational field equations.
} 
Nonlinear quantum Dirac fields were used by Heisenberg $(1953,1957)$ in his ambitious unified theory of elementary particles. They are presently the object of renewed interest since the widely known paper by Gross and Neveu (1974).

Nonlinear spinor field (NLSF) in external cosmological gravitational field was first studied by Shikin (1991). This study was extended by us for the more general case where we consider the nonlinear term as an arbitrary function of all possible invariants generated from spinor bilinear forms. In that paper, we also studied the possibility of elimination of initial singularity especially for the Kasner Universe (Rybakov et al., 1994a). For few years, we studied the behavior of self-consistent NLSF in a B-I Universe (Rybakov et al., 1994b; Saha, 2001; Saha and Shikin, 1997a) both in presence of perfect fluid and without it that was followed by Refs. (Alvarado et al., 1995a,b,c; Saha and Boyadjiev, 2004; Saha and Shikin, 1997b) where we studied the self-consistent system of interacting spinor and scalar fields. Recently, we also consider the NLSF in a Bianchi type-IV space-time as well (Saha, 2004). Moreover, a system, presenting the mixture of spinor, scalar, electromagnetic and gravitational fields, has been studied to some extent (Saha, 2005).

\subsection{Plane-Symmetric Space-Time}

In recent years, cosmological models exhibiting plane symmetry have been studied by various authors (Anguige, 2000; Chervon and Shabalkin, 2000; da Silva and Wang, 1998; Nouri-Zonoz and Tavanfar, 2001; Ori, 1998; Pradhan and Pandey, 2003; Rendall, 1995; Taruya and Nambu, 1996; Yazadjiev, 2003; Zhuravlev et al., 1997). In Ref. (Rendall, 1995) the nature of the initial singularity in spatially compact plane-symmetric scalar field cosmologies is investigated. The author shows that this singularity is crushing and velocity dominated. He also shows that the Kretschmann scalar diverges uniformly as it is approached. For a plane-symmetric cosmological model, Taruya and Nambu (1996) implemented the second-order expansion and analyzed the purely nonlinear perturbation of gravitational wave (GW). In Ref. (Anguige, 2000) the author proved the existence of a class of plane-symmetric perfect fluid cosmologies with a Kasner-like singularity of cigar type. Exact vacuum solutions of Einstein field equations corresponding to both static and stationary plane-symmetric space-times are investigated in Ref. (Nouri-Zonoz and Tavanfar, 2001). Ori constructed a class of plane-symmetric solutions possessing a curvature singularity that is null and weak, like the spacetime singularity at the Cauchy horizon of spinning (or charged) black hole (Ori, 1998). Though at the present state of evolution, the universe is spherically symmetric and the matter distribution in it is isotropic and homogeneous, in the early stage of evolution (say close to big bang singularity), neither the assumption of spherical symmetry nor of isotropy can be strictly valid. From this point of view many authors consider plane symmetry, which is less restrictive than spherical 
symmetry and provides an avenue to study inhomogeneities, since such models play an important role in understanding some essential features of the universe such as formation of galaxies during the early stage of evolution and process of homogenization. Inhomogeneous plane-symmetric model was first considered by Taub (1951, 1956). As was defined by Taub (1951), a space-time will be said to have plane symmetry if it admits the three parameter group generated by the transformation

$$
\begin{aligned}
& y^{*}=y+a, \\
& z^{*}=z+b,
\end{aligned}
$$

and

$$
\begin{gathered}
y^{*}=y \cos \theta+z \sin \theta, \\
z^{*}=-y \sin \theta+z \cos \theta .
\end{gathered}
$$

The metric of space-time admitting plane symmetry may be written as (Taub, 1956)

$$
d s^{2}=e^{2 \chi} d t^{2}-e^{2 \alpha} d x^{2}-e^{2 \beta}\left(d y^{2}+d z^{2}\right),
$$

where the speed of light $c$ is taken to be unity and $\chi, \alpha, \beta$ are functions of $x$ and $t$ alone. Note that (1.2) is one of the most general form of a universe admitting plane symmetry. Most of the authors dealing with plane-symmetric space-time consider the case when the metric functions depend on one variable, though a few consider the most general case (Taruya and Nambu, 1996; Pradhan and Pandey, 2003). Since a coordinate transformation $X=x-v t$ enables us to restore the general case, in this report we choose the metric functions to be time independent.

It should be emphasized that a space-time with plane symmetry has the following properties (Taub, 1951):

- A space-time with plane symmetry with $R_{\mu \nu}=0$ admits a coordinate system where the line element is independent of $x^{0}$, i.e., static.

- A space-time with plane symmetry with $R_{\mu \nu}=0$ admits at least one additional one-parameter group with a time-like infinitesimal generator.

- A space-time with plane symmetry with $R_{\mu \nu}=0$ and for which $R_{v \alpha \beta}^{\mu}$ is finite for all points is flat.

Thus, the space-times with plane symmetry are quite similar to those with spherical symmetry.

The universe is spherically symmetric and the matter distribution in it is on the whole isotropic and homogeneous. But during the early stages of evolution, it is unlikely that it could have had such a smoothed out picture. Hence, we consider plane symmetry which provides an opportunity for the study of inhomogeneity. In a 
recent paper (Saha and Shikin, 2004), we studied the system of minimally coupled nonlinear spinor and scalar fields in a plane-symmetric background. In that paper, the role of nonlinear spinor and scalar fields in the formation of configurations with localized energy density and limited total energy, spin and charge of the spinor field has been investigated to some extent. In the present report, we further study the system in question. We also perform some numerical study of the aforementioned system.

\section{BASIC EQUATIONS AND THEIR GENERAL SOLUTIONS}

Using the variational principle, in this section, we derive the basic equations for the corresponding spinor, scalar, and gravitational fields from the action (2.1).

We consider a system of the nonlinear spinor, scalar, and plane-symmetric gravitational fields given by the action

$$
\mathscr{A}(g ; \psi, \bar{\psi}, \varphi)=\int \mathscr{L} \sqrt{-g} d \Omega
$$

with

$$
\mathscr{L}=\mathscr{L}_{\mathrm{g}}+\mathscr{L}_{\mathrm{sp}}+\mathscr{L}_{\mathrm{sc}} .
$$

The gravitational part of the Lagrangian (2.2) $\mathscr{L}_{\mathrm{g}}$ is given by a planesymmetric metric, whereas the terms $\mathscr{L}_{\mathrm{sp}}$ and $\mathscr{L}_{\mathrm{sc}}$ describe the nonlinear spinor and scalar fields, respectively.

\subsection{Matter Field Lagrangian}

For a spinor field $\psi$, the symmetry between $\psi$ and $\bar{\psi}$ appears to demand that one should choose the symmetrized Lagrangian. Keeping this in mind, we choose the spinor field Lagrangian as

$$
\mathscr{L}_{\mathrm{sp}}=\frac{i}{2}\left[\bar{\psi} \gamma^{\mu} \nabla_{\mu} \psi-\nabla_{\mu} \bar{\psi} \gamma^{\mu} \psi\right]-m \bar{\psi} \psi+\mathscr{L}_{\mathrm{N}},
$$

with $m$ being the spinor mass. The nonlinear term $\mathscr{L}_{\mathrm{N}}$ in spinor Lagrangian describes the self-interaction of a spinor field and can be presented as some arbitrary functions of invariants generated from the real bilinear forms of a spinor field having the form

$$
\begin{aligned}
S & =\bar{\psi} \psi, \quad P=i \bar{\psi} \gamma^{5} \psi, \quad \mathrm{v}^{\mu}=\left(\bar{\psi} \gamma^{\mu} \psi\right), \\
A^{\mu} & =\left(\bar{\psi} \gamma^{5} \gamma^{\mu} \psi\right), \quad T^{\mu \nu}=\left(\bar{\psi} \sigma^{\mu \nu} \psi\right),
\end{aligned}
$$


where $\sigma^{\mu \nu}=(i / 2)\left[\gamma^{\mu} \gamma^{\nu}-\gamma^{\nu} \gamma^{\mu}\right]$. Invariants, corresponding to the bilinear forms, look like

$$
\begin{aligned}
I & =S^{2}, \quad J=P^{2}, \quad I_{\nu}=v_{\mu} v^{\mu}=\left(\bar{\psi} \gamma^{\mu} \psi\right) g_{\mu \nu}\left(\bar{\psi} \gamma^{\nu} \psi\right), \\
I_{A} & =A_{\mu} A^{\mu}=\left(\bar{\psi} \gamma^{5} \gamma^{\mu} \psi\right) g_{\mu \nu}\left(\bar{\psi} \gamma^{5} \gamma^{\nu} \psi\right) \\
I_{T} & =T_{\mu \nu} T^{\mu \nu}=\left(\bar{\psi} \sigma^{\mu \nu} \psi\right) g_{\mu \alpha} g_{\nu \beta}\left(\bar{\psi} \sigma^{\alpha \beta} \psi\right) .
\end{aligned}
$$

According to the Pauli-Fierz theorem (Berestetski et al., 1989), among the five invariants only $I$ and $J$ are independent as all other can be expressed by them: $I_{v}=-I_{A}=I+J$ and $I_{T}=I-J$. Therefore, we choose the nonlinear term $\mathscr{L}_{\mathrm{N}}=\lambda F(I, J)$, thus claiming that it describes the nonlinearity in the most general of its form. Here $\lambda$ is the constant of self-coupling.

The massless scalar field Lagrangian is chosen to be an arbitrary function of invariant $\Upsilon=\varphi_{, \alpha} \varphi^{, \alpha}$ :

$$
\mathscr{L}_{\mathrm{sc}}=\Psi(\Upsilon), \quad \Upsilon=\varphi_{, \alpha} \varphi^{, \alpha}
$$

The scalar field Lagrangian (2.4) becomes linear at $\Upsilon \rightarrow 0$, i.e.,

$$
\lim _{\Upsilon \rightarrow 0} \Psi(\Upsilon)=\frac{1}{2} \Upsilon+\cdots
$$

As a massless nonlinear scalar field Lagrangian one can choose, e.g., the BornInfeld Lagrangian (3.75).

\subsection{Gravitational Field}

As a gravitational field, we consider the plane-symmetric space-time. The gravitational part of the Lagrangian (2.2) we choose in the form

$$
\mathscr{L}_{\mathrm{g}}=\frac{R}{2 \kappa},
$$

where $R$ is the scalar curvature, $\kappa=8 \pi G$ being the Einstein's gravitational constant. The static plane-symmetric metric in our case is given in the form

$$
d s^{2}=e^{2 \chi} d t^{2}-e^{2 \alpha} d x^{2}-e^{2 \beta}\left(d y^{2}+d z^{2}\right),
$$

with $\chi, \alpha, \beta$ being the functions of the spatial variable $x$ only and obey the coordinate condition

$$
\alpha=2 \beta+\chi
$$

\subsection{Field Equation}

Let us now write the field equations corresponding to the action (2.1). 
Variation of (2.1) with respect to spinor field $\psi(\bar{\psi})$ gives nonlinear spinor field equations

$$
\begin{aligned}
& i \gamma^{\mu} \nabla_{\mu} \psi-\Phi \psi+i \mathscr{\mathscr { G }} \gamma^{5} \psi=0, \\
& i \nabla_{\mu} \bar{\psi} \gamma^{\mu}+\Phi \bar{\psi}-i \mathscr{\mathscr { S }} \bar{\psi} \gamma^{5}=0,
\end{aligned}
$$

with

$$
\Phi=m-\mathscr{D}=m-2 S \frac{\partial F}{\partial I}, \quad \mathscr{G}=2 P \frac{\partial F}{\partial J},
$$

whereas variation of (2.1) with respect to the scalar field yields the following scalar field equation

$$
\frac{1}{\sqrt{-g}} \frac{\partial}{\partial x^{\nu}}\left(\sqrt{-g} g^{\nu \mu} \frac{d \Psi}{d \Upsilon} \varphi_{, \mu}\right)=0 .
$$

Varying (2.1) with respect to metric tensor $g_{\mu \nu}$ we obtain the Einstein's field equation

$$
R_{v}^{\mu}-\frac{1}{2} \delta_{v}^{\mu} R=-\kappa T_{v}^{\mu}
$$

which in view of (2.7) and (2.8) is written as follows

$$
\begin{aligned}
& G_{0}^{0}=e^{-2 \alpha}\left(2 \beta^{\prime \prime}-2 \chi^{\prime} \beta^{\prime}-\beta^{2}\right)=-\kappa T_{0}^{0} \\
& G_{1}^{1}=e^{-2 \alpha}\left(2 \chi^{\prime} \beta^{\prime}+\beta^{\prime 2}\right)=-\kappa T_{1}^{1} \\
& G_{2}^{2}=e^{-2 \alpha}\left(\beta^{\prime \prime}+\chi^{\prime \prime}-2 \chi^{\prime} \beta^{\prime}-\beta^{\prime 2}\right)=-\kappa T_{2}^{2} \\
& G_{3}^{3}=G_{2}^{2}, \quad T_{3}^{3}=T_{2}^{2} .
\end{aligned}
$$

Here prime denotes differentiation with respect to $x$ and $T_{v}^{\mu}$ is the energymomentum tensor of the spinor and scalar fields

$$
T_{\mu}^{v}=T_{\mathrm{sp} \mu}^{v}+T_{\mathrm{sc} \mu}^{v} .
$$

The energy-momentum tensor of the spinor field is

$$
T_{\mathrm{sp} \mu}^{\rho}=\frac{i}{4} g^{\rho \nu}\left(\bar{\psi} \gamma_{\mu} \nabla_{\nu} \psi+\bar{\psi} \gamma_{\nu} \nabla_{\mu} \psi-\nabla_{\mu} \bar{\psi} \gamma_{\nu} \psi-\nabla_{\nu} \bar{\psi} \gamma_{\mu} \psi\right)-\delta_{\mu}^{\rho} \mathscr{L}_{\mathrm{sp}},
$$

where $\mathscr{L}_{\text {sp }}$ with respect to (2.9) takes the form

$$
\mathscr{L}_{\mathrm{sp}}=-\frac{1}{2}\left(\bar{\psi} \frac{\partial F}{\partial \bar{\psi}}+\frac{\partial F}{\partial \psi} \psi\right)-F
$$

and the energy-momentum tensor of the scalar field is

$$
T_{\mathrm{sc} \mu}^{\nu}=2 \frac{d \Psi}{d \Upsilon} \varphi_{, \mu} \varphi^{, \nu}-\delta_{\mu}^{\nu} \Psi, \quad \Upsilon=-\left(\varphi^{\prime}\right)^{2} e^{-2 \alpha}, \quad \varphi^{\prime}=\frac{d \varphi}{d x} .
$$


In (2.9) and (2.14) $\nabla_{\mu}$ denotes the covariant derivative of spinor, having the form (Brill and Wheeler, 1957; Zhelnorovich, 1982)

$$
\nabla_{\mu} \psi=\frac{\partial \psi}{\partial x^{\mu}}-\Gamma_{\mu} \psi
$$

where $\Gamma_{\mu}(x)$ are spinor affine connection matrices. $\gamma$ matrices in the earlier equations are connected with the flat space-time Dirac matrices $\bar{\gamma}$ in the following way

$$
g_{\mu \nu}(x)=e_{\mu}^{a}(x) e_{\nu}^{b}(x) \eta_{a b}, \quad \gamma_{\mu}(x)=e_{\mu}^{a}(x) \bar{\gamma}_{a},
$$

where $\eta_{a b}=\operatorname{diag}(1,-1,-1,-1)$ and $e_{\mu}^{a}$ is a set of tetrad 4-vectors. Using (2.18) we obtain

$$
\gamma^{0}(x)=e^{-\chi} \bar{\gamma}^{0}, \quad \gamma^{1}(x)=e^{-\alpha} \bar{\gamma}^{1}, \quad \gamma^{2}(x)=e^{-\beta} \bar{\gamma}^{2}, \quad \gamma^{3}(x)=e^{-\beta} \bar{\gamma}^{3} .
$$

From

$$
\Gamma_{\mu}(x)=\frac{1}{4} g_{\rho \sigma}(x)\left(\partial_{\mu} e_{\delta}^{b} e_{b}^{\rho}-\Gamma_{\mu \delta}^{\rho}\right) \gamma^{\sigma} \gamma^{\delta}
$$

one finds

$$
\begin{aligned}
& \Gamma_{0}=-\frac{1}{2} \bar{\gamma}^{0} \bar{\gamma}^{1} e^{-2 \beta} \chi^{\prime}, \quad \Gamma_{1}=0, \quad \Gamma_{2}=\frac{1}{2} \bar{\gamma}^{2} \bar{\gamma}^{1} e^{-(\chi+\beta)} \beta^{\prime}, \\
& \Gamma_{3}=\frac{1}{2} \bar{\gamma}^{3} \bar{\gamma}^{1} e^{-(\chi+\beta)} \beta^{\prime} .
\end{aligned}
$$

Flat space-time matrices $\bar{\gamma}$ we will choose in the form, given in (Bogoliubov and Shirkov, 1976):

$$
\begin{array}{rlrl}
\bar{\gamma}^{0} & =\left(\begin{array}{rrrr}
1 & 0 & 0 & 0 \\
0 & 1 & 0 & 0 \\
0 & 0 & -1 & 0 \\
0 & 0 & 0 & -1
\end{array}\right), & \bar{\gamma}^{1}=\left(\begin{array}{rrrrr}
0 & 0 & 0 & 1 \\
0 & 0 & 1 & 0 \\
0 & -1 & 0 & 0 \\
-1 & 0 & 0 & 0
\end{array}\right), \\
\bar{\gamma}^{2}=\left(\begin{array}{rrrr}
0 & 0 & 0 & -i \\
0 & 0 & i & 0 \\
0 & i & 0 & 0 \\
-i & 0 & 0 & 0
\end{array}\right), & \bar{\gamma}^{3}=\left(\begin{array}{rrrr}
0 & 0 & 1 & 0 \\
0 & 0 & 0 & -1 \\
-1 & 0 & 0 & 0 \\
0 & 1 & 0 & 0
\end{array}\right) .
\end{array}
$$

Defining $\gamma^{5}$ as follows,

$$
\begin{aligned}
& \gamma^{5}=-\frac{i}{4} E_{\mu \nu \sigma \rho} \gamma^{\mu} \gamma^{\nu} \gamma^{\sigma} \gamma^{\rho}, \quad E_{\mu \nu \sigma \rho}=\sqrt{-g} \varepsilon_{\mu \nu \sigma \rho}, \quad \varepsilon_{0123}=1, \\
& \gamma^{5}=-i \sqrt{-g} \gamma^{0} \gamma^{1} \gamma^{2} \gamma^{3}=-i \bar{\gamma}^{0} \bar{\gamma}^{1} \bar{\gamma}^{2} \bar{\gamma}^{3}=\bar{\gamma}^{5}
\end{aligned}
$$


we obtain

$$
\bar{\gamma}^{5}=\left(\begin{array}{rccr}
0 & 0 & -1 & 0 \\
0 & 0 & 0 & -1 \\
-1 & 0 & 0 & 0 \\
0 & -1 & 0 & 0
\end{array}\right) .
$$

The scalar field Equation (2.10) has the solution

$$
\frac{d \Psi}{d \Upsilon} \varphi^{\prime}=\varphi_{0}, \quad \varphi_{0}=\text { constant }
$$

The equality (2.22) for a given $\Psi(\Upsilon)$ is an algebraic equation for $\varphi^{\prime}$ that is to be defined through metric function $e^{\alpha(x)}$.

We will consider the spinor field to be the function of the spatial coordinate $x$ only $[\psi=\psi(x)]$. Using (2.17), (2.19), and (2.21) we find

$$
\gamma^{\mu} \Gamma_{\mu}=-\frac{1}{2} e^{-\alpha} \alpha^{\prime} \bar{\gamma}^{1}
$$

Then taking into account (2.23) we rewrite the spinor field Equation (2.9a) as

$$
i \bar{\gamma}^{1}\left(\frac{\partial}{\partial x}+\frac{\alpha^{\prime}}{2}\right) \psi+i e^{\alpha} \Phi \psi+e^{\alpha} \mathscr{G} \gamma^{5} \psi=0 .
$$

Further setting $V(x)=e^{\alpha / 2} \psi(x)$ with

$$
V(x)=\left(\begin{array}{l}
V_{1}(x) \\
V_{2}(x) \\
V_{3}(x) \\
V_{4}(x)
\end{array}\right)
$$

for the components of spinor field from (2.24) one deduces the following system of equations:

$$
\begin{gathered}
V_{4}^{\prime}+i e^{\alpha} \Phi V_{1}-e^{\alpha} \mathscr{G} V_{3}=0, \\
V_{3}^{\prime}+i e^{\alpha} \Phi V_{2}-e^{\alpha} \mathscr{G} V_{4}=0, \\
V_{2}^{\prime}-i e^{\alpha} \Phi V_{3}+e^{\alpha} \mathscr{G} V_{1}=0, \\
V_{1}^{\prime}-i e^{\alpha} \Phi V_{4}+e^{\alpha} \mathscr{G} V_{2}=0 .
\end{gathered}
$$

As one sees, Equation (2.25) gives following relations

$$
V_{1}^{2}-V_{2}^{2}-V_{3}^{2}+V_{4}^{2}=\text { constant. }
$$

From (2.9) one can write the equations for $S=\bar{\psi} \psi, P=i \bar{\psi} \gamma^{5} \psi$, and $A=\bar{\psi} \bar{\gamma}^{5} \bar{\gamma}^{1} \psi$

$$
S^{\prime}+\alpha^{\prime} S+2 e^{\alpha} \mathscr{\mathscr { g }} A=0
$$




$$
\begin{aligned}
P^{\prime}+\alpha^{\prime} P+2 e^{\alpha} \Phi A & =0, \\
A^{\prime}+\alpha^{\prime} A+2 e^{\alpha} \Phi P+2 e^{\alpha} \mathscr{G} S & =0 .
\end{aligned}
$$

Note that $A$ in (2.27) is indeed the pseudo-vector $A^{1}$. Here, for simplicity, we use the notation $A$. Further defining $S_{0}=S e^{\alpha}, P_{0}=P e^{\alpha}, A_{0}=A e^{\alpha}$, from (2.27) we find

$$
\begin{aligned}
S_{0}^{\prime}+2 e^{\alpha} \mathscr{G} A_{0} & =0, \\
P_{0}^{\prime}+2 e^{\alpha} \Phi A_{0} & =0, \\
A_{0}^{\prime}+2 e^{\alpha} \Phi P_{0}+2 e^{\alpha} \mathscr{G} S_{0} & =0 .
\end{aligned}
$$

The system (2.27) admits the following solution

$$
S^{2}+P^{2}-A^{2}=C_{0} e^{-2 \alpha}, \quad C_{0}=\text { constant. }
$$

Beside this from the system (2.28) follows

$$
\Phi S_{0}^{\prime}-\mathscr{G} P_{0}^{\prime}=0 .
$$

Let us now solve the Einstein equations. To do it we first write the expression for the components of the energy-momentum tensor explicitly. Using the property of flat space-time Dirac matrices and the explicit form of covariant derivative $\nabla_{\mu}$, for the spinor field one finds

$$
T_{\mathrm{sp} 1}^{1}=m S-F(I, J), \quad T_{\mathrm{sp} 0}^{0}=T_{\mathrm{sp} 2}^{2}=T_{\mathrm{sp} 3}^{3}=\mathscr{C} S+\mathscr{g} P-F(I, J) .
$$

On the other hand, taking into account that the scalar field $\varphi$ is also a function of $x$ only $[\varphi=\varphi(x)]$ for the scalar field one obtains

$$
T_{\mathrm{sc} 1}^{1}=2 \Upsilon \frac{d \Psi}{d \Upsilon}-\Psi(\Upsilon), \quad T_{\mathrm{sc} 0}^{0}=T_{\mathrm{sc} 2}^{2}=T_{\mathrm{sc} 3}^{3}=-\Psi(\Upsilon) .
$$

In view of $T_{0}^{0}=T_{2}^{2}$, subtraction of Einstein equations (2.12a) and (2.12c) leads to the equation

$$
\beta^{\prime \prime}-\chi^{\prime \prime}=0
$$

with the solution

$$
\beta(x)=\chi(x)+B x,
$$

where $B$ is the integration constant. The second constant has been chosen to be trivial, since it acts on the scale of $Y$ - and $Z$-axes only. On the account of (2.33) from (2.8) one obtains

$$
\beta^{\prime \prime}=\frac{1}{3} \alpha^{\prime \prime}, \quad \chi^{\prime \prime}=\frac{1}{3} \alpha^{\prime \prime} .
$$


Solutions to Equation (2.35) together with (2.8) and (2.34) lead to the following expression for $\beta(x)$ and $\chi(x)$

$$
\beta(x)=\frac{1}{3}(\alpha(x)+B x), \quad \chi(x)=\frac{1}{3}(\alpha(x)-2 B x) .
$$

Equation (2.12b), being the first integral of (2.12a) and (2.12c), is a first-order differential equation. Inserting $\beta$ and $\gamma$ from (2.36) and $T_{1}^{1}$ in account of (2.13), (2.31), and (2.32) into (2.12b) for $\alpha$ one gets

$$
\alpha^{\prime 2}-B^{2}=-3 \kappa e^{2 \alpha}\left[m S-F(I, J)+2 \Upsilon \frac{d \Psi}{d \Upsilon}-\Psi(\Upsilon)\right] .
$$

As one sees from (2.27) and (2.29), the invariants are the functions of $\alpha$, so is the right-hand side of (2.37), hence can be solved in quadrature. In the sections to follow, we analyze Equation (2.37) in detail given the concrete form of the spinor and scalar field Lagrangian.

\subsection{Physical Observable Values}

To build a descriptive picture of any physical theory we need to express the results through real physical values, which can be measured experimentally. In General Relativity, where we are dealing with the objects in 4-D space-time, the problem of defining the physical observable values is not a trivial. A mathematical apparatus to calculate the physical observable values in 4-D pseudo-Riemannian space was first introduced by Zelmanov and is referred to as theory of chronometric invariants (Mitskievich, 1969; Rabounski and Borisova, 2001; Zelmanov and Agakov, 1989). By chronometric-invariant values it is understood that physical observable values in accompanying frame should be invariant with respect to transformation of time.

Let us study this point in detail. Consider two system of coordinates $x^{i}$ and $x^{\prime} i$. These two systems are said to be related to the one and the same system of reference if they obey the following relations

$$
\frac{\partial x^{\prime} i}{\partial x^{0}}=0, \quad \frac{\partial x^{i}}{\partial x^{\prime} 0}=0 .
$$

The earlier going conditions mean, the systems of coordinates belonging to the one and the same system of reference are stationary to each other. On the other hand, if two system of coordinates move relative to each other they belong to different system of references. Then the coordinate transformation that leaves the two system of coordinates in the one and the same system of reference can be written as a system of two transformations realized together: chronometric transformations and 3-D transformation

$$
x^{\prime 0}=x^{\prime 0}\left(x^{0}, x^{1}, x^{2}, x^{3}\right),
$$




$$
x^{\prime i}=x^{\prime i}\left(x^{1}, x^{2}, x^{3}\right) .
$$

Quantities invariant under the group of transformations (2.39) are called to be chronometric-invariant quantities. According to Zelmanov theorem chronometrically invariant (physical observable) projections of 4-D vector $Q^{\alpha}$ are (Rabounski and Borisova, 2001).

$$
u^{\alpha} Q_{\alpha}=\frac{Q_{0}}{\sqrt{g_{00}}}, \quad P_{\alpha}^{i} Q^{\alpha}=Q^{i},
$$

where $u^{\alpha}$ is the 4-D velocity with $u_{\alpha} u^{\alpha}=1$ and $P_{\alpha \beta}$ is the projection operator:

$$
\begin{aligned}
P_{\alpha \beta} & =g_{\alpha \beta}-u_{\alpha} u_{\beta} \\
P^{\alpha \beta} & =g^{\alpha \beta}-u^{\alpha} u^{\beta} \\
P_{\beta}^{\alpha} & =\delta_{\beta}^{\alpha}-u^{\alpha} u_{\beta} \\
P_{\lambda}^{\alpha} P_{\beta}^{\lambda} & =P_{\beta}^{\alpha} .
\end{aligned}
$$

As it was mentioned earlier, one of the purposes of this paper is to study the role of the nonlinear spinor and scalar fields in the formation of configurations with localized energy density and limited total energy, spin, and charge of the spinor field. In doing so, we first define the spinor current and spin tensor.

The components of spinor current can be written in the form (Bogoliubov and Shirkov, 1976):

$$
j^{\mu}=\bar{\psi} \gamma^{\mu} \psi .
$$

Taking into account that $\bar{\psi}=\psi^{\dagger} \bar{\gamma}^{0}$, where $\psi^{\dagger}=\left(\psi_{1}^{*}, \psi_{2}^{*}, \psi_{3}^{*}, \psi_{4}^{*}\right)$ and $\psi_{j}=$ $e^{-\alpha / 2} V_{j}, j=1,2,3,4$ for the components of spinor current we write

$$
\begin{aligned}
& j^{0}=\left[V_{1}^{*} V_{1}+V_{2}^{*} V_{2}+V_{3}^{*} V_{3}+V_{4}^{*} V_{4}\right] e^{-(\alpha+\chi)}, \\
& j^{1}=\left[V_{1}^{*} V_{4}+V_{2}^{*} V_{3}+V_{3}^{*} V_{2}+V_{4}^{*} V_{1}\right] e^{-2 \alpha}, \\
& j^{2}=-i\left[V_{1}^{*} V_{4}-V_{2}^{*} V_{3}+V_{3}^{*} V_{2}-V_{4}^{*} V_{1}\right] e^{-(\alpha+\beta)}, \\
& j^{3}=\left[V_{1}^{*} V_{3}-V_{2}^{*} V_{4}+V_{3}^{*} V_{1}-V_{4}^{*} V_{2}\right] e^{-(\alpha+\beta)} .
\end{aligned}
$$

Since we consider the field configuration to be static, the spatial components of spinor current vanishes, i.e.,

$$
j^{1}=0, \quad j^{2}=0, \quad j^{3}=0 .
$$

This supposition gives additional relation between the constant of integration. The component $j^{0}$ defines the charge density of spinor field that has the following chronometric-invariant form

$$
\rho=\left(j_{0} \cdot j^{0}\right)^{1 / 2} .
$$


Note that the definition of chronometric-invariant values adopted here differs from one suggested by Zelmanov. In our case we just underline the fact that the experimentalist measures namely $\rho$, not $j^{0}$. So being the physical observable value $\rho$ can be termed as chronometric invariant. The total charge of spinor field is defined as

$$
Q=\int_{-\infty}^{\infty} \int_{-\infty}^{\infty} \int_{-\infty}^{\infty} \rho \sqrt{-{ }^{3} g} d x d y d z
$$

Since $\rho=\rho(x)$, i.e., matter distribution takes place along $x$-axis only, for the charge $Q$ to make any sense we should integrate it for any finite range by $y$ and $z$ and then normalize it to unity. Sometimes $Q$ is defined as

$$
Q=\frac{\int_{-\infty}^{\infty} \int_{-\infty}^{\infty} \int_{-\infty}^{\infty} \rho \sqrt{-{ }^{3} g} d x d y d z}{\int_{-\infty}^{\infty} \int_{-\infty}^{\infty} d y d z}
$$

In what follows we perform integration by $y$ and $z$ in the limit $(0,1)$ and define the total charge (normalized) as

$$
Q=\int_{-\infty}^{\infty} \rho \sqrt{-{ }^{3} g} d x .
$$

Following (Bogoliubov and Shirkov, 1976) we define the spin tensor as

$$
S^{\mu \nu, \epsilon}=\frac{1}{4} \bar{\psi}\left\{\gamma^{\epsilon} \sigma^{\mu \nu}+\sigma^{\mu \nu} \gamma^{\epsilon}\right\} \psi .
$$

Let us now write the components $S^{i k, 0}(i, k=1,2,3)$, defining the spatial density of spin vector, explicitly. From (2.49) we have

$$
S^{i j, 0}=\frac{1}{4} \bar{\psi}\left\{\gamma^{0} \sigma^{i j}+\sigma^{i j} \gamma^{0}\right\} \psi=\frac{1}{2} \bar{\psi} \gamma^{0} \sigma^{i j} \psi
$$

that defines the projection of spin vector on $k$-axis. Here $i, j, k$ takes the value $1,2,3$ and $i \neq j \neq k$. Thus, for the projection of spin vectors on the $X-, Y$-, and $Z$-axis we find

$$
\begin{aligned}
& S^{23,0}=\left[V_{1}^{*} V_{2}+V_{2}^{*} V_{1}+V_{3}^{*} V_{4}+V_{4}^{*} V_{3}\right] e^{-\alpha-2 \beta-\chi}, \\
& S^{31,0}=\left[V_{1}^{*} V_{2}-V_{2}^{*} V_{1}+V_{3}^{*} V_{4}-V_{4}^{*} V_{3}\right] e^{-2 \alpha-\beta-\chi}, \\
& S^{12,0}=\left[V_{1}^{*} V_{1}-V_{2}^{*} V_{2}+V_{3}^{*} V_{3}-V_{4}^{*} V_{4}\right] e^{-2 \alpha-\beta-\chi}
\end{aligned}
$$

The chronometric-invariant spin tensor takes the form

$$
S_{\mathrm{ch}}^{i j, 0}=\left(S_{i j, 0} S^{i j, 0}\right)^{1 / 2},
$$


and the projection of the spin vector on $k$-axis is defined by

$$
S_{k}=\int_{-\infty}^{\infty} S_{\mathrm{ch}}^{i j, 0} \sqrt{-{ }^{3} g} d x
$$

Once the solution to the spinor and gravitational field equations are obtained, using the equalities written earlier one immediately finds the physical quantities such as total charge, spin, etc. for the corresponding nonlinear spinor field.

\section{EXACT SOLUTIONS TO THE BASIC EQUATIONS AND THEIR PHYSICAL INTERPRETATIONS}

In this section, we write some exact solutions to the basic equations derived in the foregoing section for a concrete choice of the nonlinear terms in the spinor and scalar field Lagrangian. We also give physical interpretations to the corresponding solutions.

\subsection{Case with Linear Spinor and Scalar Fields}

Let us consider the self-consistent system of linear spinor and massless scalar field equations. By doing so we can compare the results obtained with those of the self-consistent system of nonlinear spinor and scalar field equations, hence clarify the role of nonlinearity of the fields in question in the formation of regular localized solutions such as static solitary wave or soliton (Adomou and Shikin, 1998a; Shikin, 1995).

In this case for the scalar field, we have $\Psi(\Upsilon)=\frac{1}{2} \Upsilon$. Inserting this into (2.22) we obtain

$$
\varphi^{\prime}(x)=\varphi_{0}
$$

From (2.32) in account of (3.1) we get

$$
-T_{\mathrm{sc} 1}^{1}=T_{\mathrm{sc} 0}^{0}=T_{\mathrm{sc} 2}^{2}=T_{\mathrm{sc} 3}^{3}=-\frac{1}{2} \Upsilon=\frac{1}{2} \varphi_{0}^{2} e^{-2 \alpha} .
$$

On the other hand, for the linear spinor field we have

$$
T_{\mathrm{sp} 1}^{1}=m S, \quad T_{\mathrm{sp} 0}^{0}=T_{\mathrm{sp} 2}^{2}=T_{\mathrm{sp} 3}^{3}=0 .
$$

As one can easily verify, for the linear spinor field Equation (2.27a) results

$$
S=C_{0} e^{-\alpha}
$$


Taking this relation into account and the fact that $\alpha^{\prime}(x)=-\frac{1}{S} \frac{d S}{d x}$ from (2.37), we write

$$
\int \frac{d S}{\sqrt{(1+\bar{\kappa} / 2) B^{2} S^{2}-3 \kappa C_{0}^{2} S}}=x, \quad \bar{\kappa}=3 \kappa \varphi_{0}^{2} / B^{2} .
$$

Denoting $M^{2}=3 \kappa C_{0}^{2}$ and $H^{2}=B^{2}(1+\bar{\kappa} / 2)$ from (3.5) we obtain

$$
\frac{1}{H} \ln \left|\frac{H^{2} S-M^{2} / 2}{H}+\sqrt{H^{2} S^{2}-M^{2} S}\right|=x .
$$

In Fig. 1 we show the behavior of the metric functions $\alpha, \beta$, and $\chi$ with respect to the spatial parameter $x$. Note that the initial growth of $\beta$ takes place only for the linear case for the given set of parameters chosen here.

Let us now go back to spinor field Equation (2.25). Setting $V_{j}(x)=U_{j}(S)$, $j=1,2,3,4$ and taking into account that in this case $\Phi=m$ and $\mathcal{G}=0$, for $U_{j}(S)$ we obtain

$$
\begin{aligned}
& \frac{d U_{4}}{d S}+i \mathscr{F}(S) U_{1}=0, \\
& \frac{d U_{3}}{d S}+i \mathscr{F}(S) U_{2}=0,
\end{aligned}
$$

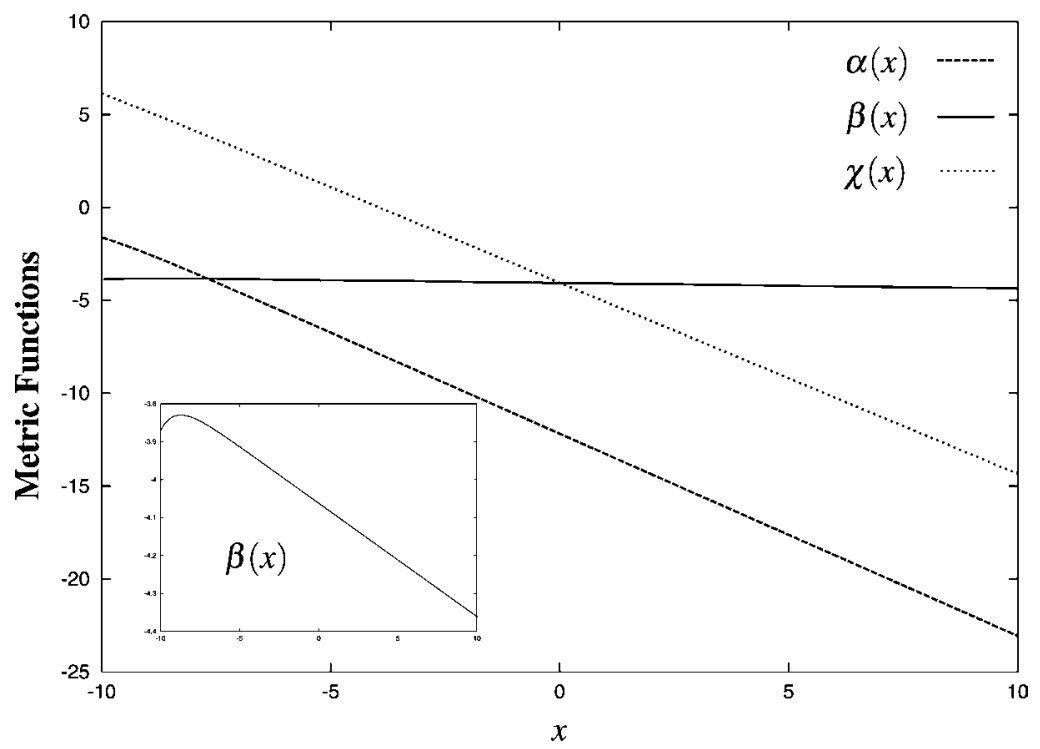

Fig. 1. View of the metric functions $\alpha(x), \beta(x)$, and $\chi(x)$ with the parameters $\kappa=1, C_{0}=1$, $\varphi_{0}=0.5$, and $B=1$ in case of linear spinor and scalar fields. 


$$
\begin{aligned}
& \frac{d U_{2}}{d S}-i \mathscr{F}(S) U_{3}=0, \\
& \frac{d U_{1}}{d S}-i \mathscr{F}(S) U_{4}=0,
\end{aligned}
$$

with

$$
\mathscr{F}(S)=m C_{0} / S \sqrt{H^{2} S^{2}-M^{2} S} .
$$

Equations (3.7a) and (3.7d) can be combined together to get

$$
U_{1}^{2}+U_{4}^{2}=C_{14}^{2}, \quad C_{14}^{2}=\text { constant. }
$$

A similar relation yields from Equations (3.7b) and (3.7c) for $U_{2}$ and $U_{3}$. Taking this into account we finally find

$$
\begin{array}{ll}
U_{1}=i C_{14} \sinh \left(f_{1}(S)\right), & U_{4}=C_{14} \cosh \left(f_{1}(S)\right), \\
U_{2}=i C_{23} \sinh \left(f_{1}(S)\right), & U_{3}=C_{23} \cosh \left(f_{1}(S)\right) .
\end{array}
$$

Here

$$
f_{1}(S)=\int \mathscr{F}(S) d S=\frac{2 m C_{0} \sqrt{H^{2} S^{2}-M^{2} S}}{M^{2} S}=\frac{2 m C_{0} \sqrt{H^{2}-M^{2} / S}}{M^{2}} .
$$

The positivity of the radical in (3.5) imposes $S>M^{2} / H^{2}>0$. On the other hand, $f_{1}$ increases with the growth of $S$ and takes the maximum value $\left.f_{1 \max }\right|_{S \rightarrow \infty} \approx\left(2 m / 3 \kappa C_{0}\right) \sqrt{B^{2}+3 \kappa \varphi_{0}^{2}}$.

For the scalar field energy density, we find

$$
T_{\mathrm{sc} 0}^{0}(x)=\frac{1}{2} \varphi_{0}^{2} e^{-2 \alpha}=\frac{\varphi_{0}^{2} S^{2}}{2 C_{0}^{2}} .
$$

From (3.6) we see that $S$ grows exponentially with $x$, therefore, the scalar field energy density is not localized which follows from (3.10).

Let us consider the case when the scalar field possesses negative energy density. Then we have $\Psi(\Upsilon)=-(1 / 2) \Upsilon$ and

$$
-T_{\mathrm{sc} 1}^{1}=T_{\mathrm{sc} 0}^{0}=T_{\mathrm{sc} 2}^{2}=T_{\mathrm{sc} 3}^{3}=\frac{1}{2} \Upsilon=-\frac{1}{2} \varphi_{0}^{2} e^{-2 \alpha}
$$

Then for $S$ we get

$$
\int \frac{d S}{\sqrt{(1-\bar{\kappa} / 2) B^{2} S^{2}-3 \kappa C_{0}^{2} S}}=x .
$$

As one sees, the field system considered here is physically realizable if and only if $1-\bar{\kappa} / 2>0$, i.e., the scalar charge $\left|\varphi_{0}\right|<\sqrt{2 / 3 \kappa} B$. Moreover, in the specific case with $B=0$, independent to the quantity of scalar charge $\varphi_{0}$, the existence of 
scalar field with negative energy density in general relativity is impossible even in absence of linear spinor field.

Inserting (3.9) into (2.43) one finds the components of the spinor current:

$$
\begin{aligned}
& j^{0}=\left(C_{14}^{2}+C_{23}^{2}\right)\left(\sinh ^{2}\left(f_{1}(S)\right)+\cosh ^{2}\left(f_{1}(S)\right)\right) e^{-(\alpha+\chi)}, \\
& j^{1}=0, \\
& j^{2}=2\left(C_{23}^{2}-C_{14}^{2}\right) \sinh ^{2}\left(f_{1}(S)\right) \cosh ^{2}\left(f_{1}(S)\right) e^{-(\alpha+\beta)}, \\
& j^{3}=0 .
\end{aligned}
$$

In view of (2.44), from (3.13) follows that $C_{14}=C_{23}$. For the projections of spin vector, one then finds

$$
\begin{aligned}
& S^{23,0}=2 C_{14}^{2}\left(\sinh ^{2}\left(f_{1}(S)\right)+\cosh ^{2}\left(f_{1}(S)\right)\right) e^{-\alpha-2 \beta-\chi}, \\
& S^{31,0}=0, \\
& S^{12,0}=0 .
\end{aligned}
$$

In view of (2.45) and (2.52), from (2.48) and (2.53) in case of linear spinor and scalar fields one then finds the following expressions for the total charge $Q$ and the projection of spin vector along $X$-axis:

$$
Q=4 C_{14}^{4} \int_{-\infty}^{\infty}\left[\sinh ^{2}\left(f_{1}(S)\right)+\cosh ^{2}\left(f_{1}(S)\right)\right]^{2} d x
$$

and

$$
S_{x}=4 C_{14}^{4} \int_{-\infty}^{\infty}\left[\sinh ^{2}\left(f_{1}(S)\right)+\cosh ^{2}\left(f_{1}(S)\right)\right]^{2} d x
$$

As one sees from (3.15) and (3.16), given the suitable value of the constants $B, C_{0}, \varphi_{0}$, and $\kappa$ the total charge of the system may be finite. Thus, we see that, in case of linear spinor and scalar fields with minimal coupling both charge and spin of spinor field are limited. The energy density of the system, in view of (3.3) is defined by the contribution of scalar field only:

$$
T_{0}^{0}(x)=T_{\mathrm{sc} 0}^{0}(x)=\frac{\varphi_{0}^{2} S^{2}}{2 C_{0}^{2}} .
$$

From (3.17) follows that, the energy density of the system is not localized and the total energy of the system $E=\int_{-\infty}^{\infty} T_{0}^{0} \sqrt{-3} g d x$ is not finite.

Figures 2 and 3 show the behavior of $S$ with respect to $x$ for the system with different types of nonlinearities. As one sees, $S(x)$ for the system with nonlinear 


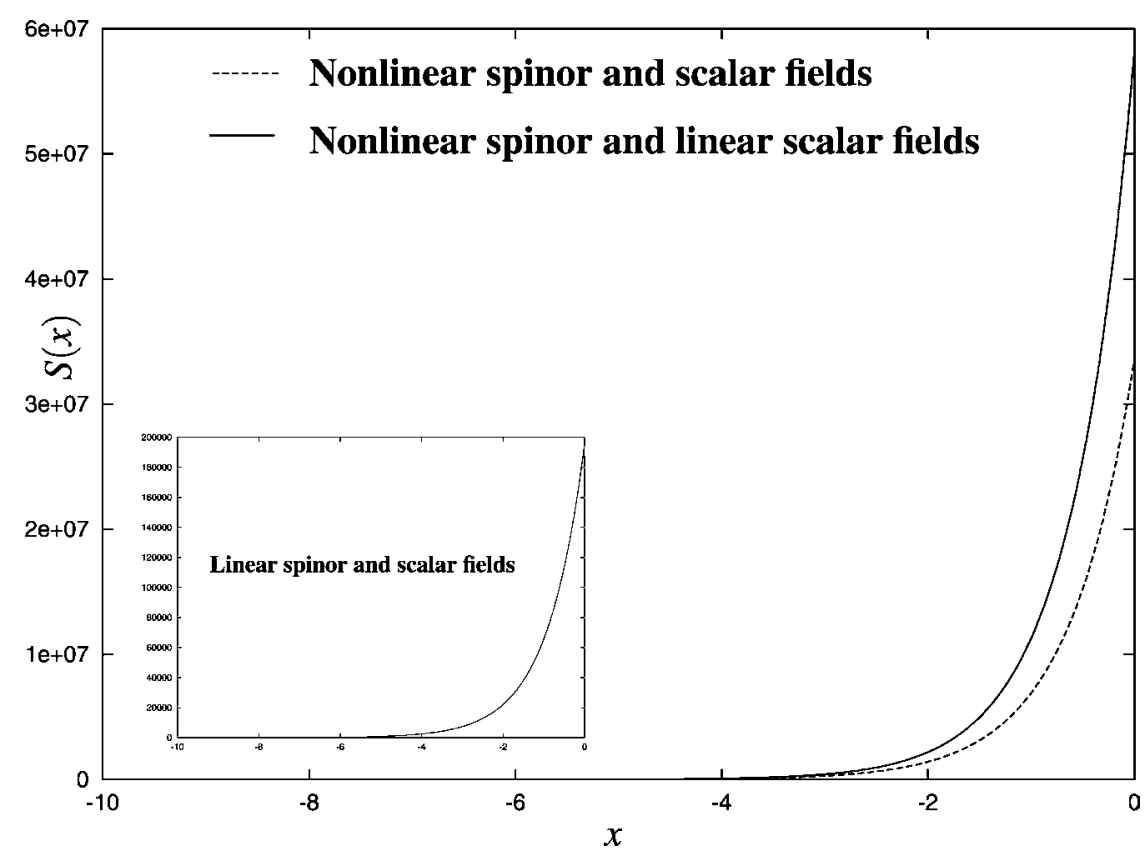

Fig. 2. View of $S(x)$ for three different cases with $S_{\text {in }}=5$. It clearly shows the role of the spinor field nonlinearity in the growth of $S(x)$.

spinor term grows rather rapid. Introduction of the nonlinear scalar field, though slightly, slows the growth of $S(x)$.

\subsection{Nonlinear Spinor and Linear Scalar Fields}

Case 1. $(F=F(I))$ Let us consider the case when the nonlinear term in spinor field Lagrangian is a function of $I(S)$ only, that leads to $\mathcal{G}=0$. From (2.27) as in case of linear spinor field we find $S=C_{0} e^{-\alpha(x)}$. In case of linear scalar filed, i.e., for $\Psi(\Upsilon)=(a / 2) \Upsilon$, where $a= \pm 1$ we have $\Upsilon=-\varphi_{0}^{2} e^{-2 \alpha}$. Taking it into mind and proceeding as in foregoing subsection, for $S$ from (2.37) we write

$$
\frac{d S}{d x}= \pm \mathcal{L}(S), \quad \mathcal{L}(S)=\sqrt{B^{2} S^{2}-3 \kappa C_{0}^{2}\left[m S-F(S)-a \varphi_{0}^{2} S^{2} /\left(2 C_{0}^{2}\right)\right]}
$$

with the solution

$$
\int \frac{d S}{\mathcal{L}(S)}= \pm\left(x+x_{0}\right) .
$$

Given the concrete form of the functions $F(S)$, from (3.19) yields $S$, hence $\alpha, \beta, \chi$. 


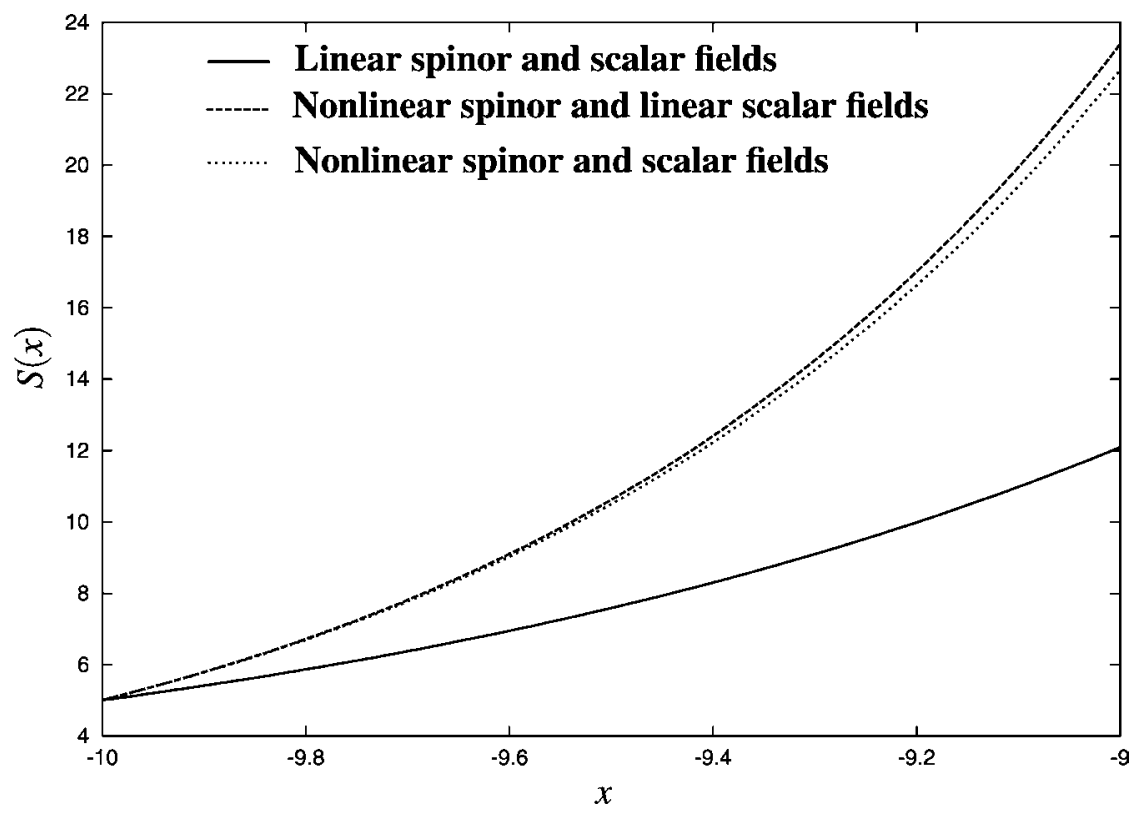

Fig. 3. This figure shows the behavior of $S(x)$ illustrated in Fig. 2 near the starting point.

Let us now go back to spinor field Equation (2.25). Setting $V_{j}(x)=U_{j}(S)$, $j=1,2,3,4$ and taking into account that in this case $\mathcal{G}=0$, for $U_{j}(S)$ we obtain

$$
\begin{aligned}
& \frac{d U_{4}}{d S}+i \mathscr{F}(S) U_{1}=0, \\
& \frac{d U_{3}}{d S}+i \mathscr{F}(S) U_{2}=0, \\
& \frac{d U_{2}}{d S}-i \mathscr{F}(S) U_{3}=0, \\
& \frac{d U_{1}}{d S}-i \mathscr{F}(S) U_{4}=0,
\end{aligned}
$$

with $\mathscr{F}(S)=\Phi \mathcal{L}(S) C_{0} / S$. Differentiating (3.20a) with respect to $S$ and inserting (3.20d) into it for $U_{4}$ we find

$$
\frac{d^{2} U_{4}}{d S^{2}}-\frac{1}{\mathscr{F}} \frac{d \mathscr{F}}{d S} \frac{d U_{4}}{d S}-\mathscr{\mathscr { H }}^{2} U_{4}=0
$$

that transforms to

$$
\frac{1}{\mathscr{F}} \frac{d}{d S}\left(\frac{1}{\mathscr{\mathscr { F }}} \frac{d U_{4}}{d S}\right)-U_{4}=0
$$


with the first integral

$$
\frac{d U_{4}}{d S}= \pm \sqrt{U_{4}^{2}+C_{1}} \cdot \mathscr{F}(S), \quad C_{1}=\text { constant }
$$

For $C_{1}=a_{1}^{2}>0$ from (3.23) we obtain

$$
U_{4}(S)=a_{1} \sinh N_{1}(S), \quad N_{1}= \pm \int \mathscr{F}(S) d S+R_{1}, \quad R_{1}=\text { constant }
$$

whereas for $C_{1}=-b_{1}^{2}<0$ from (3.23) we obtain

$$
U_{4}(S)=a_{1} \cosh N_{1}(S)
$$

Inserting (3.24) and (3.25) into (3.20d) one finds

$$
U_{1}(S)=i a_{1} \cosh N_{1}(S), \quad U_{1}(S)=i b_{1} \sinh N_{1}(S) .
$$

Analogically, for $U_{2}$ and $U_{3}$ we obtain

$$
U_{3}(S)=a_{2} \sinh N_{2}(S), \quad U_{3}(S)=b_{2} \cosh N_{2}(S) .
$$

and

$$
U_{2}(S)=i a_{2} \cosh N_{2}(S), \quad U_{2}(S)=i b_{2} \sinh N_{2}(S) .
$$

where $N_{2}= \pm \int \mathscr{F}(S) d S+R_{2}$ and $a_{2}, b_{2}$, and $R_{2}$ are the integration constants. Thus, we find the general solutions to the spinor field Equation (3.20) containing four arbitrary constants.

Using the solutions obtained, from (2.43) we find the components of spinor current

$$
\begin{aligned}
j^{0} & =\left[a_{1}^{2} \cosh \left(2 N_{1}(S)\right)+a_{2}^{2} \cosh \left(2 N_{2}(S)\right)\right] e^{-(\alpha+\chi)}, \\
j^{1} & =0 \\
j^{2} & =-\left[a_{1}^{2} \sinh \left(2 N_{1}(S)\right)-a_{2}^{2} \sinh \left(2 N_{2}(S)\right)\right] e^{-(\alpha+\beta)}, \\
j^{3} & =0 .
\end{aligned}
$$

The supposition (2.44) leads to the following relations between the constants: $a_{1}=a_{2}=a$ and $R_{1}=R_{2}=R$, since $N_{1}(S)=N_{2}(S)=N(S)$. The chronometricinvariant form of the charge density and the total charge of spinor field are

$$
\begin{aligned}
& \rho=2 a^{2} \cosh (2 N(S)) e^{-\alpha}, \\
& Q=2 a^{2} \int_{-\infty}^{\infty} \cosh (2 N(S)) e^{\alpha-\chi} d x .
\end{aligned}
$$

From (2.50) we find

$$
S^{12,0}=0, \quad S^{13,0}=0, \quad S^{23,0}=a^{2} \cosh (2 N(S)) e^{-2 \alpha} .
$$


Thus, the only nontrivial component of the spin tensor is $S^{23,0}$ that defines the projection of spin vector on $X$-axis. From (2.52) we write the chronometricinvariant spin tensor

$$
S_{\mathrm{ch}}^{23,0}=a^{2} \cosh (2 N(S)) e^{-\alpha},
$$

and the projection of the spin vector on $X$-axis

$$
S_{1}=a^{2} \int_{-\infty}^{\infty} \cosh (2 N(S)) e^{\alpha-\chi} d x .
$$

(In (2.53), as well as in (2.48) integrations by $y$ and $z$ are performed in the limit $(0,1))$. Note that the integrants both in (3.31) and (3.34) coincide.

Let us now analyze the result obtained choosing the nonlinear term in the form $F(I)=\lambda S^{n}=\lambda I^{n / 2}$ with $n \geq 2$ and $\lambda$ is the parameter of nonlinearity. For $n=2$ we have Heisenberg-Ivanenko type nonlinear spinor field equation (Ivanenko, 1959)

$$
i e^{-\alpha} \bar{\gamma}^{1}\left(\partial_{x}+\frac{1}{2} \alpha^{\prime}\right) \psi-m \psi+2 \lambda(\bar{\psi} \psi) \psi=0 .
$$

Setting $F=S^{2}$ into (3.19) we come to the expression for $S$ that is similar to that for linear case with

$$
H^{2} \rightarrow H_{1}^{2}=B^{2}+3 \kappa \lambda C_{0}+3 \kappa \varphi_{0}^{2} / 2 .
$$

The behavior of the metric functions, namely, $\exp (2 \alpha(x)), \exp (2 \beta(x))$, and $\exp (2 \chi(x))$, has been illustrated in Fig. 4.

Let us write the functions $\psi_{j}$ explicitly. In this case, we have

$$
\mathscr{F}(S)=m\left(C_{0}-2 \lambda S\right) / S \sqrt{H_{1}^{2} S^{2}-M^{2} S},
$$

and

$$
N_{1,2}(x)=\left(2 H_{1} / 3 \kappa C_{0}\right) \tanh \left(\bar{H}_{1} x\right)-2 \lambda C_{0} x+R_{1,2}, \quad \bar{H}_{1}=H_{1} / 2 .
$$

We can then finally write

$$
\begin{aligned}
& \psi_{1,2}(x)=i a_{1,2} \frac{\sqrt{3 \kappa m C_{0}}}{H_{1}} \cosh \left(\bar{H}_{1} x\right) \cosh N_{1,2}(x), \\
& \psi_{3,4}(x)=i a_{2,1} \frac{\sqrt{3 \kappa m C_{0}}}{H_{1}} \cosh \left(\bar{H}_{1} x\right) \cosh N_{2,1}(x) .
\end{aligned}
$$

Let us consider the energy-density distribution of the field system:

$$
T_{0}^{0}=\left(\lambda+\frac{1}{2} \frac{\varphi_{0}^{2}}{C_{0}^{2}}\right) \frac{M^{4}}{H_{1}^{4}} \cosh ^{4}\left(\bar{H}_{1} x\right) .
$$




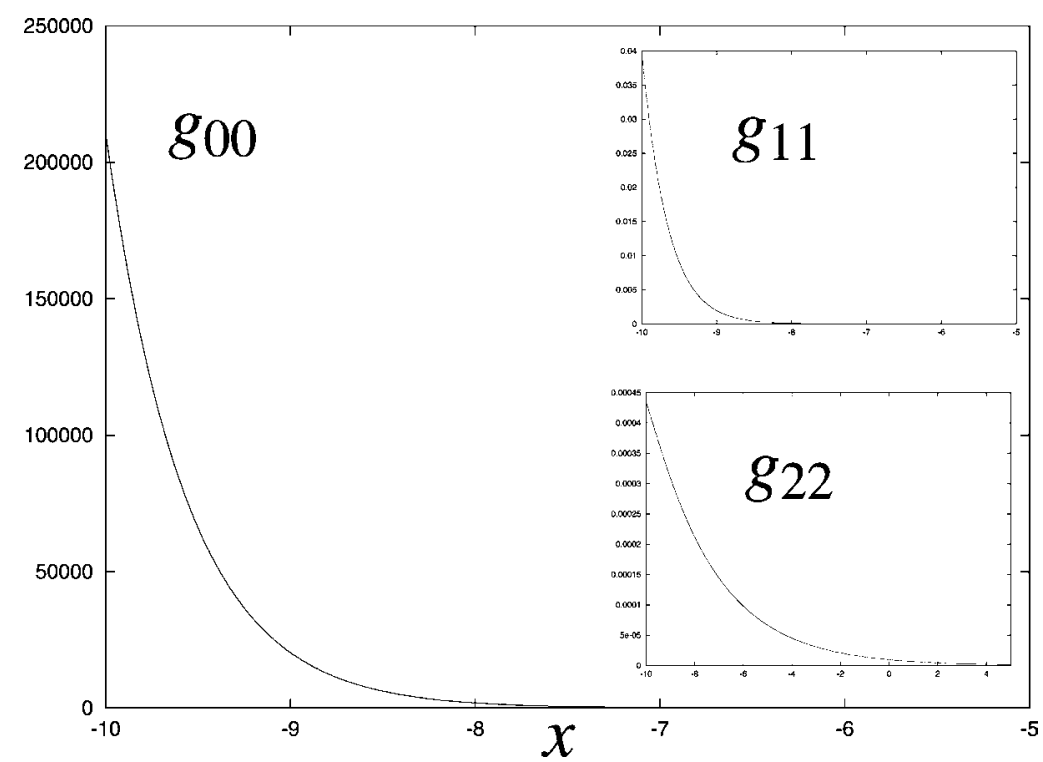

Fig. 4. View of $g_{00}(x), g_{11}(x)$, and $g_{22}(x)$ in case of nonlinear spinor and linear scalar fields. Here as a nonlinear spinor field we consider the Heisenberg-Ivanenko case.

From (3.38) follows that, the energy density of the system is not localized and the total energy of the system $E=\int_{-\infty}^{\infty} T_{0}^{0} \sqrt{-{ }^{3} g} d x$ is not finite. Note that, the energy density of the system can be trivial, if

$$
\lambda+\frac{1}{2} \frac{\varphi_{0}^{2}}{C_{0}^{2}}=0 .
$$

It is possible, if and only if the sign of energy density of spinor and scalar fields are different.

Let us write the total charge of the system.

$$
\begin{aligned}
Q= & 2 a^{2} \int_{-\infty}^{\infty} \cosh \left[\frac{4 H_{1}}{3 \kappa C_{0}} \tanh \left(\bar{H}_{1} x\right)-4 \lambda C_{0} x+2 R\right] \\
& \times\left(\frac{C_{0} H_{1}^{2}}{M^{2} \cosh ^{2}\left(\bar{H}_{1} x\right)}\right)^{3 / 2} e^{2 B x / 3} d x .
\end{aligned}
$$

If $12 \lambda^{2} C_{0}^{2}+\lambda C_{0}\left(4 B-\kappa C_{0}\right)-\kappa \varphi_{0}^{2} / 2<0$, the integral (3.40) converges, that means the possibility of existence of finite charge and spin of the system. 
In case of $n>2$, the energy density of the system in question is

$$
T_{0}^{0}=\lambda(n-1) S^{n}+\frac{1}{2} \frac{\varphi_{0}^{2}}{C_{0}^{2}} S^{2}
$$

which shows that the regular solutions with localized energy density exists if and only if $S=\bar{\psi} \psi$ is a continuous and limited function and $\lim _{x \rightarrow \pm \infty} S(x) \rightarrow 0$. The condition, when $S$ possesses the properties mentioned earlier is

$$
\int \frac{d S}{\sqrt{(1+\bar{\kappa} / 2) B^{2} S^{2}-3 \kappa C_{0}^{2}\left(m S-\lambda S^{n}\right)}}=x .
$$

As one sees from (3.42), for $m \neq 0$ at no value of $x S$ becomes trivial, since as $S \rightarrow 0$, the denominator of the integrant beginning from some finite value of $S$ becomes imaginary. It means that for $S(x)$ to be trivial at spatial infinity $(x \rightarrow \infty)$, it is necessary to choose massless spinor field setting $m=0$ in (3.42). Note that, in the unified nonlinear spinor theory of Heisenberg, the massive term is absent, and according to Heisenberg, the particle mass should be obtained as a result of quantization of spinor prematter (Heisenberg, 1968). It should be emphasized that in the nonlinear generalization of classical field equations, the massive term does not possess the significance that it possesses in the linear one, as it by no means defines total energy (or mass) of the nonlinear field system (Schweber, 1961). Thus, without losing the generality, we can consider massless spinor field putting $m=0$. Note that in the sections to follow where we consider the nonlinear spinor term as $F=P^{n}$, or $F=\left(K_{ \pm}\right)^{n}$ with $K_{ \pm}=(I \pm J)$, we will study the massless spinor field only.

From (3.42) for $m=0, \lambda>0$, and $n>2$ for $S(x)$ we obtain

$$
S(x)=\left[-H_{1} / \sqrt{3 \kappa \lambda C_{0}^{2}\left(\zeta^{2}-1\right)}\right]^{2 /(n-2)}, \quad \zeta=\cosh \left[(n-2) \bar{H}_{1} x\right]
$$

from which follows that $\lim _{x \rightarrow 0}|S(x)| \rightarrow \infty$. It means that $T_{0}^{0}(x)$ is not bounded at $x=0$ and the initial system of equations does not possess solutions with localized energy density.

If we set in (3.42) $m=0, \lambda=-\Lambda^{2}<0$ and $n>2$, then for $S$ we obtain

$$
S(x)=\left[H_{1} / \sqrt{3 \kappa \lambda C_{0}^{2}} \zeta\right]^{2 /(n-2)}
$$

It is seen from (3.44) that $S(x)$ has maximum at $x=0$ and $\lim _{x \rightarrow \pm \infty} S(x) \rightarrow 0$. Corresponding graphical view of $S(x)$ is given in Fig. 5 . 


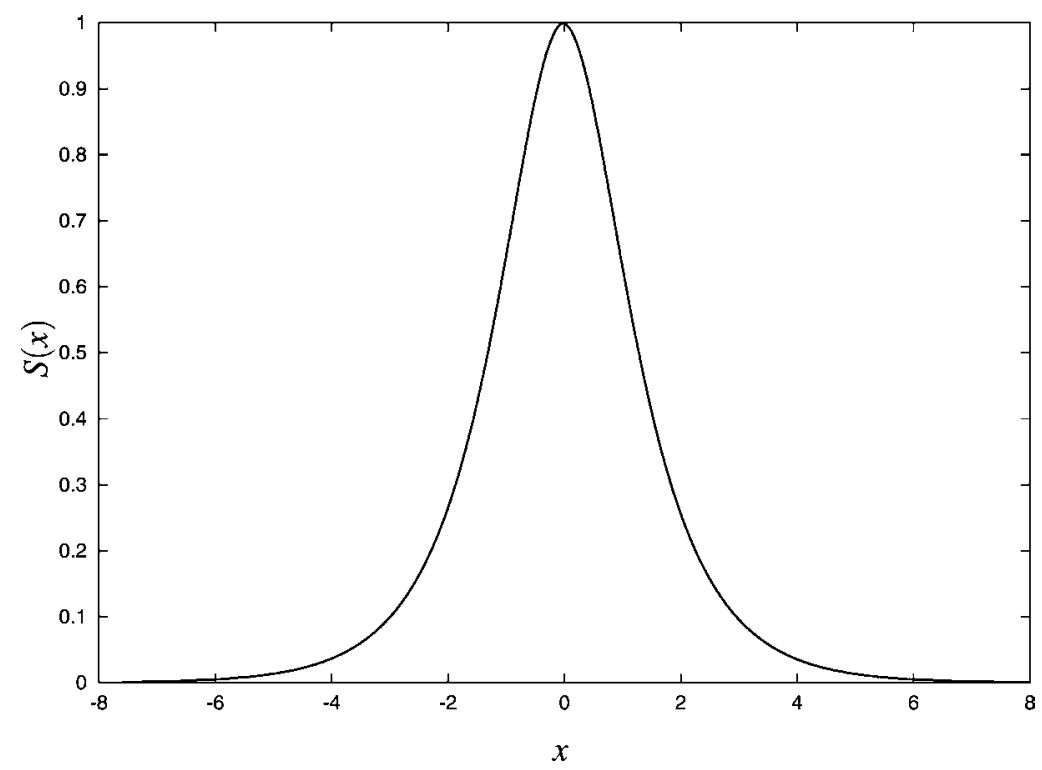

Fig. 5. View of $S(x)$ in case of a nonlinear spinor field with $n=4$. As one sees, in the case considered the function $S(x)$ has its maximum at $x=0$.

For energy density we have

$$
T_{0}^{0}=-\Lambda^{2}(n-1) S^{n}+\frac{1}{2} \frac{\varphi_{0}^{2}}{C_{0}^{2}} S^{2},
$$

where $S$ is defined by (3.44). In view of $S$ it follows that $T_{0}^{0}(x)$ is an alternating function.

Let us find the condition when the total energy of the system is bound

$$
E=\int_{-\infty}^{\infty} T_{0}^{0} \sqrt{-{ }^{3} g} d x<\infty .
$$

For this we write the integrant of (3.46)

$$
\varepsilon(x)=T_{0}^{0} \sqrt{-{ }^{3} g}=C_{0}^{5 / 3}\left[\frac{\varphi_{0}^{2}}{2 C_{0}^{2}}-\frac{(n-1) H_{1}^{2} \zeta^{2}}{3 \kappa \lambda C_{0}^{2}}\right]\left[\frac{H_{1}^{2} \zeta}{3 \kappa \Lambda^{2} C_{0}^{2}}\right]^{1 / 3(n-2)} e^{2 B x / 3} .
$$

From (3.47) follows that $\lim _{x \rightarrow-\infty} \varepsilon(x) \rightarrow 0$ for any value of the parameters, while $\lim _{x \rightarrow+\infty} \varepsilon(x) \rightarrow 0$ iff $H>2 B$ or $\kappa \varphi_{0}^{2}>2 B^{2}$. Note that in this case the contribution 
of scalar field to the total energy is positive and finite:

$$
T_{\mathrm{sc} 0}^{0}=\frac{\varphi_{0}^{2}}{2 C_{0}^{2}} S^{2}, \quad E_{\mathrm{sc}}=\int_{-\infty}^{\infty} T_{\mathrm{sc} 0}^{0} \sqrt{-{ }^{3} g} d x<\infty .
$$

Note that in the case considered the scalar field is linear and massless. As far as in absence of spinor field energy density of the linear scalar field is not localized and the total energy in not finite, in the case considered the properties of the field configurations are defined by those of nonlinear spinor field. The contribution of nonlinear spinor field to the total energy is negative. Moreover, it remains finite even in absence of scalar field for $n>2$ (Adomou and Shikin, 1998b).

The components of spinor field in this case have the form

$$
\begin{aligned}
& \psi_{1,2}(x)=i a_{1,2} E(x) \cosh N_{1,2}(x), \\
& \psi_{3,4}(x)=a_{2,1} E(x) \sinh N_{2,1}(x),
\end{aligned}
$$

where

$$
E(x)=\left(1 / \sqrt{C_{0}}\right)\left[H_{1} / \sqrt{3 \kappa \Lambda^{2} C_{0}^{2}} \zeta\right]^{1 /(n-2)}
$$

and

$$
N_{1,2}(x)=-\frac{2 n H_{1} \sqrt{\zeta^{2}-1}}{3 \kappa C_{0}(n-2) \zeta}+R_{1,2} .
$$

For the solutions obtained we write the chronometric-invariant charge density of the spinor field $\rho$ :

$$
\rho(x)=\frac{2 a^{2}}{C_{0}} \cosh \left\{-\frac{4 n H_{1} \sqrt{\zeta^{2}-1}}{3 \kappa C_{0}(n-2) \zeta}+2 R\right\}\left\{\frac{H_{1}^{2}}{3 \kappa \Lambda^{2} C_{0}^{2} \zeta^{2}}\right\}^{1 /(n-2)} .
$$

As one sees from (3.50), the charge density is localized, since $\lim _{x \rightarrow \pm \infty} \rho(x) \rightarrow 0$. Nevertheless, the charge density of the spinor field, coming to unit invariant volume $\rho \sqrt{-3} g$, is not localized:

$$
\rho \sqrt{-{ }^{3} g}=2 a^{2} \cosh [2 N(x)] e^{\alpha-\gamma}=2 a^{2} \cosh [2 N(x)]\left(C_{0} / S\right)^{2 / 3} e^{2 B x / 3} .
$$

It leads to the fact that the total charge of the spinor field is not bounded as well. As far as the expression for chronometric-invariant tensor of spin (3.33) coincides with that of $\rho(x) / 2$, the conclusions made for $\rho(x)$ and $Q$ will be valid for the spin tensor $S_{\mathrm{ch}}^{23,0}$ and projection of spin vector on $X$-axis $S_{1}$, i.e., $S_{\mathrm{ch}}^{23,0}$ is localized and $S_{1}$ is unlimited.

The solution obtained describes the configuration of nonlinear spinor and linear scalar fields with localized energy density but with the metric that is singular 
at spatial infinity, as in this case

$$
e^{2 \alpha}=\left(C_{0} / S\right)^{2}=\left.C_{0}^{2}\left\{\frac{3 \kappa \Lambda C_{0}^{2} \zeta}{H_{1}^{2}}\right\}^{2 /(n-2)}\right|_{x \rightarrow \pm \infty} \rightarrow \infty
$$

Let us consider the massless spinor field with

$$
F=-\Lambda^{2} S^{-v}, \quad v=\text { constant }>0 .
$$

In this case, the energy density of the system of nonlinear spinor and linear scalar fields with minimal coupling takes the form

$$
T_{0}^{0}=\Lambda^{2}(v+1) S^{-v}+\frac{\varphi_{0}^{2}}{2 C_{0}^{2}} S^{2} .
$$

For $S$ in this case we get

$$
\int \frac{d S}{\sqrt{(1+\bar{\kappa} / 2) B^{2} S^{2}-3 \kappa C_{0}^{2} \Lambda^{2} S^{-v}}}=x
$$

with the solution

$$
S(x)=\left[\frac{3 \kappa \Lambda^{2} C_{0}^{2}}{H_{1}^{2}} \zeta_{1}^{2}\right]^{1 /(v+2)}, \quad \zeta_{1}=\cosh \left[(v+2) \bar{H}_{1} x\right] .
$$

For energy density in this case we have

$$
T_{0}^{0}(x)=\Lambda^{2}(v+1)\left[\frac{H_{1}^{2}}{3 \kappa C_{0}^{2} \Lambda^{2} \zeta_{1}^{2}}\right]^{\nu /(v+2)}+\frac{\varphi_{0}^{2}}{2 C_{0}^{2}}\left[\frac{3 \kappa C_{0}^{2} \Lambda^{2} \zeta_{1}^{2}}{H_{1}^{2}}\right]^{2 /(v+2)}
$$

It follows from (3.57) that the contribution of the spinor field in the energy density is localized while for the scalar field it is not the case.

The energy density distribution of the field system, coming to unit invariant volume is

$$
\begin{aligned}
\varepsilon(x) & =T_{0}^{0} \sqrt{-{ }^{3} g}=\left[\Lambda^{2}(\nu+1) S^{-v}+\frac{\varphi_{0}^{2}}{2 C_{0}^{2}} S^{2}\right] e^{2 \alpha-\gamma} \\
& =\left\{\frac{H_{1}^{2}(\nu+1)}{3 \kappa \zeta_{1}^{2}}+\frac{\varphi_{0}^{2}}{2}\right\}\left\{\frac{H_{1}^{2}}{3 \kappa C_{0}^{2} \Lambda^{2} \zeta_{1}^{2}}\right\}^{1 / 3(\nu+2)} e^{2 B x / 3} .
\end{aligned}
$$

As one sees from (3.58) $\varepsilon(x)$ is a localized function, i.e., $\lim _{x \rightarrow \pm \infty} \varepsilon(x) \rightarrow 0$, if $H>2 B$ or $\kappa \varphi_{0}^{2}>2 B^{2}$. In this case the total energy is also finite.

The components of spinor field in this case have the form

$$
\begin{aligned}
& \psi_{1,2}(x)=i a_{1,2} E(x) \cosh N_{1,2}(x), \\
& \psi_{3,4}(x)=a_{2,1} E(x) \sinh N_{2,1}(x),
\end{aligned}
$$


where

$$
E(x)=\left(1 / \sqrt{C_{0}}\right)\left[\frac{\sqrt{3 \kappa \Lambda^{2} C_{0}^{2}}}{H_{1}^{2}} \zeta_{1}\right]^{1 /(v+2)}
$$

and

$$
N_{1,2}(x)=-\frac{2 H v \sqrt{\zeta_{1}^{2}-1}}{3 \kappa C_{0}(\nu+2) \zeta_{1}}+R_{1,2} .
$$

The chronometric-invariant charge density of the spinor field coming to unit invariant volume with $a_{1}=a_{2}=a$ and $N_{1}=N_{2}$ reads

$$
\begin{aligned}
\rho \sqrt{-^{3} g}= & 2 a^{2} \cosh [2 N(x)] e^{\alpha-\gamma}=2 a^{2}\left(C_{0}\right)^{2 / 3} \cosh \\
& \times\left\{2 R-\frac{4 H_{1} v \sqrt{\zeta_{1}^{2}-1}}{3 \kappa C_{0}(v+2) \zeta_{1}}\right\}\left\{\frac{H_{1}^{2}}{3 \kappa C_{0}^{2} \Lambda^{2} \zeta_{1}^{2}}\right\}^{2 / 3(v+2)} e^{2 B x / 3} .
\end{aligned}
$$

It follows from (3.60) that $\varrho \sqrt{-{ }^{3} g}$ is a localized function and the total charge $Q$ is finite. The spin of spinor field is limited as well.

Case 2. $(F=F(J))$ Here we consider the massless spinor field with the nonlinearity $F=F(J)$. In this case from (2.27b) immediately follows

$$
P=D_{0} e^{-\alpha(x)}, \quad D_{0}=\text { constant. }
$$

From (2.25) we now have

$$
\begin{aligned}
& V_{4}^{\prime}-e^{\alpha} \mathscr{G} V_{3}=0, \\
& V_{3}^{\prime}-e^{\alpha} \mathscr{G} V_{4}=0, \\
& V_{2}^{\prime}+e^{\alpha} \mathscr{G} V_{1}=0, \\
& V_{1}^{\prime}+e^{\alpha} \mathscr{G} V_{2}=0,
\end{aligned}
$$

with the solutions

$$
\begin{aligned}
V_{1} & =C_{1} \sinh \left[-\mathcal{A}+C_{2}\right] \\
V_{2} & =C_{1} \cosh \left[-\mathcal{A}+C_{2}\right] \\
V_{3} & =C_{3} \sinh \left[\mathcal{A}+C_{4}\right] \\
V_{4} & =C_{3} \cosh \left[\mathcal{A}+C_{4}\right]
\end{aligned}
$$

with $C_{1}, C_{2}, C_{3}$, and $C_{3}$ being the constant of integration and $\mathcal{A}=\int e^{\alpha} \mathscr{C} d x$. 
Using the solutions obtained, from (2.43) we now find the components of spinor current

$$
\begin{aligned}
& j^{0}=\left[C_{1}^{2} \cosh \left[2\left(-\mathcal{A}+C_{2}\right)\right]+C_{3}^{2} \cosh \left[2\left(\mathcal{A}+C_{4}\right)\right]\right] e^{-(\alpha+\chi)}, \\
& j^{1}=\left[2 C_{1} C_{3} \sinh \left(C_{2}+C_{4}\right)\right] e^{-2 \alpha} \\
& j^{2}=0 \\
& j^{3}=-\left[2 C_{1} C_{3} \cosh \left[2 \mathcal{A}-C_{2}+C_{4}\right)\right] e^{-(\alpha+\beta)} .
\end{aligned}
$$

The supposition (2.44) that the spatial components of the spinor current are trivial leads at least one of the constants $\left(C_{1}, C_{3}\right)$ to be zero. Let us set $C_{1}=0$. The chronometric-invariant form of the charge density and the total charge of spinor field are

$$
\begin{gathered}
\rho=C_{3}^{2} \cosh \left[2\left(\mathcal{A}+C_{4}\right)\right] e^{-\alpha}, \\
Q=C_{3}^{2} \int_{-\infty}^{\infty} \cosh \left[2\left(\mathcal{A}+C_{4}\right)\right] e^{\alpha-\chi} d x .
\end{gathered}
$$

From (2.50) we find

$$
S^{12,0}=-C_{3}^{2} e^{-(2 \alpha+\beta+\chi)}, \quad S^{31,0}=0, \quad S^{23,0}=C_{3}^{2} \sinh \left[2\left(\mathcal{A}+C_{4}\right)\right] e^{-2 \alpha} .
$$

Thus, in this case we have two nontrivial components of the spin tensor $S^{23,0}$ and $S^{12,0}$ that define the projections of spin vector on $X$ - and $Z$-axis, respectively. From (2.52) we write the chronometric-invariant spin tensor

$$
\begin{aligned}
& S_{\mathrm{ch}}^{23,0}=C_{3}^{2} \sinh \left[2\left(\mathcal{A}+C_{4}\right)\right] e^{-\alpha}, \\
& S_{\mathrm{ch}}^{23,0}=C_{3}^{2} e^{-\alpha}
\end{aligned}
$$

and the projections of the spin vector on $X$ - and $Z$-axes are

$$
\begin{aligned}
& S_{1}=C_{3}^{2} \int_{-\infty}^{\infty} \sinh \left[2\left(\mathcal{A}+C_{4}\right)\right] e^{\alpha-\chi} d x, \\
& S_{3}=C_{3}^{2} \int_{-\infty}^{\infty} e^{\alpha-\chi} d x .
\end{aligned}
$$

Note that the equation for $\alpha$, therefore for $P$ will be the same as in previous case (i.e., for $S$ with $m=0$ ):

$$
\int \frac{d P}{\sqrt{B^{2} P^{2}-3 \kappa D_{0}^{2}\left(-F(P)-a \varphi_{0}^{2} P^{2} /\left(2 D_{0}^{2}\right)\right)}}= \pm\left(x+x_{0}\right) \text {. }
$$


In the subsection to follow we study Equation (3.70) together with (3.18) numerically.

Case 3. $(F=F(I \pm J)) \quad$ Let us now consider the case with $F=F\left(K_{ \pm}\right)$where $K_{ \pm}=I \pm J$. As in case 2, we consider the massless spinor field. In this case we then have $\mathscr{D}=2 S F_{ \pm}$and $\mathscr{G}= \pm 2 P F_{ \pm}$, where we denote $F_{ \pm}=d F / d K_{ \pm}$. Taking into account that in case of $m=0, \Phi=-\mathscr{Q}$, from (2.30) we immediately find

$$
I \pm J=K_{ \pm}=K_{0} e^{-2 \alpha}
$$

with $K_{0}$ being some arbitrary constant. The equation for $\alpha$ in this case coincides with the one given for $P$ :

$$
\int \frac{d K_{ \pm}}{\sqrt{B^{2} K_{ \pm}^{2}-3 \kappa K_{0} K_{ \pm}\left(-F\left(K_{ \pm}\right)-a \varphi_{0}^{2} K_{ \pm} /\left(2 K_{0}\right)\right)}}= \pm 2\left(x+x_{0}\right) .
$$

Note that, the relation (3.71) can be written as $S_{0}^{2} \pm P_{0}^{2}=K_{0}$, i.e., we can present $S_{0}$ and $P_{0}$ as some trigonometric functions such as:

$$
\begin{array}{ll}
S_{0}=\sin (\xi), & P_{0}=\cos (\xi), \\
S_{0}=\sinh (\xi), & P_{0}=\cosh (\xi),
\end{array}
$$

with $\xi=\sqrt{K_{0}} \int F_{ \pm} d x$. After a little manipulation, one can also write the solutions to the spinor field equations in terms of hypergeometric function.

\subsection{Nonlinear Scalar Field in Absence of Spinor Field}

Let us consider the system of gravitational and nonlinear scalar fields. As a nonlinear scalar field Lagrangian we choose Born-Infeld theory, given by the Lagrangian (Adomou and Shikin, 1998a)

$$
\Psi(\Upsilon)=-\frac{1}{\sigma}(1-\sqrt{1+\sigma \Upsilon}),
$$

with $\Upsilon=\varphi_{, \alpha} \varphi^{, \alpha}$ and $\sigma$ is the parameter of nonlinearity. From (3.75) we also have

$$
\lim _{\sigma \rightarrow 0} \Psi(\Upsilon)=\frac{1}{2} \Upsilon \cdots
$$

Inserting (3.75) into (2.22) for the scalar field we obtain the equation

$$
\varphi^{\prime}(x)=\frac{\varphi_{0}}{\sqrt{1+\sigma \varphi_{0}^{2} e^{-2 \alpha(x)}}},
$$


that gives

$$
\Upsilon=-\left(\varphi^{\prime}\right)^{2} e^{-2 \alpha}=-\frac{\varphi_{0}^{2} e^{-2 \alpha(x)}}{1+\sigma \varphi_{0}^{2} e^{-2 \alpha(x)}} .
$$

From (3.77) follows that $\left.\varphi^{\prime}\right|_{\sigma=0}=\varphi_{0}$.

For the case considered in this section we have

$$
T_{\mathrm{sc} 0}^{0}=T_{\mathrm{sc} 2}^{2}=T_{\mathrm{sc} 3}^{3}=-\Psi(\Upsilon)=\frac{1}{\sigma}\left(1-1 / \sqrt{1+\sigma \varphi_{0}^{2} e^{-2 \alpha(x)}}\right),
$$

and

$$
T_{\mathrm{sc} 1}^{1}=2 \Upsilon \frac{d \Psi}{d \Upsilon}-\Psi=\frac{1}{\sigma}\left(1-\sqrt{1+\sigma \varphi_{0}^{2} e^{-2 \alpha(x)}}\right) .
$$

Putting (3.80) into (2.37), in account of $m=0$ and $F(I, J) \equiv 0$ for $\alpha$ we find

$$
\alpha^{\prime}= \pm \sqrt{B^{2}-\frac{3 \kappa}{\sigma} e^{2 \alpha}\left(1-\sqrt{1+\sigma \varphi_{0}^{2} e^{-2 \alpha(x)}}\right)} .
$$

From (3.81) one finds

$$
\begin{aligned}
& \int \frac{d \alpha}{\sqrt{B^{2}-\frac{3 \kappa}{\sigma} e^{2 \alpha}\left(1-\sqrt{1+\sigma \varphi_{0}^{2} e^{-2 \alpha(x)}}\right)}}=-\frac{2}{B} \ln \left|\xi+\sqrt{\bar{\kappa}+\xi^{2}}\right| \\
& +\frac{1}{B \sqrt{1+\bar{\kappa} / 2}}\left[\ln \left|\sqrt{2} B \sqrt{\bar{\kappa}+\xi^{2}}+\sqrt{2} B \sqrt{1+\bar{\kappa} \xi / 2}\right|\right. \\
& \left.-\ln \left|\sqrt{3 \kappa \varphi_{0}^{2}\left(\xi^{2}-2\right)}\right|\right]=x,
\end{aligned}
$$

with $\xi^{2}=1+\sqrt{1+\sigma \varphi_{0}^{2} e^{-2 \alpha(x)}}$. As one sees from (3.82)

$$
\begin{aligned}
\left.e^{2 \alpha(x)}\right|_{x \rightarrow+\infty} & \approx \frac{\sigma \varphi_{0}^{2}}{2} e^{2 \sqrt{1+\bar{\kappa} / 2} B x} \rightarrow \infty, \\
\left.e^{2 \alpha(x)}\right|_{x \rightarrow-\infty} & \approx \frac{\sigma \varphi_{0}^{2}}{2} e^{2 B x} \rightarrow 0 .
\end{aligned}
$$

Let us study the energy density distribution of nonlinear scalar field. From (3.79) we find

$$
\left.T_{\mathrm{sc} 0}^{0}(x)\right|_{x=-\infty}=\frac{1}{\sigma},\left.\quad T_{\mathrm{sc} 0}^{0}(x)\right|_{x=\infty}=0
$$


which shows that the energy density of the scalar field is not localized. Nevertheless, the energy density on unit invariant volume is localized if $\kappa \varphi_{0}^{2}>2 B^{2}$ :

$$
\varepsilon(x)=T_{\mathrm{sc} 0}^{0} \sqrt{-{ }^{3} g}=\left.\frac{1}{\sigma}\left(1-\frac{1}{1+\sigma \varphi_{0}^{2} e^{-2 \alpha}}\right) e^{5 \alpha / 3+2 B x / 3}\right|_{x \rightarrow \pm \infty} \rightarrow 0 .
$$

In this case the total energy of the scalar field is also bound. From (3.78) in account of (3.83) and (3.84) we also have

$$
\left.\Upsilon(x)\right|_{x=-\infty}=-\frac{1}{\sigma},\left.\quad \Upsilon(x)\right|_{x=+\infty}=0,
$$

showing that $\Upsilon(x)$ is kink like.

\subsection{Nonlinear Spinor and Nonlinear Scalar Field}

Finally, we consider the self-consistent system of nonlinear spinor and scalar fields. We choose the self-action of the spinor field as $F=\lambda S^{n}, n>2$, where as the scalar field is taken in the form (3.75). Using the line of reasoning mentioned earlier, we conclude that the spinor field considered here should be massless. Taking into account that $e^{-2 \alpha}=S^{2} / C_{0}^{2}$ for $S$ we write

$$
\int \frac{d S}{\sqrt{B^{2} S^{2}+3 \kappa C_{0}^{2}\left[\lambda S^{n}+\left(\sqrt{1+\sigma \varphi_{0}^{2} S^{2} / C_{0}^{2}}-1\right) / \sigma\right]}}=x .
$$

From (3.88) one estimates

$$
\left.S(x)\right|_{x \rightarrow 0} \sim \frac{1}{x^{2 /(n-2)}} \rightarrow \infty .
$$

On the other hand, for the energy density we have

$$
T_{0}^{0}=\lambda(n-1) S^{n}+\frac{1}{\sigma}\left(1-1 / \sqrt{1+\sigma \varphi_{0}^{2} S^{2} / C_{0}^{2}}\right)
$$

that states that for $T_{0}^{0}$ to be localized $S$ should be localized too and $\lim _{x \rightarrow \pm \infty} S(x) \rightarrow 0$. Hence, from (3.89) we conclude that $S(x)$ is singular and energy density in unlimited at $x=0$.

For $\lambda=-\Lambda^{2}$ and $n>2$ we have

$$
\int \frac{d S}{\sqrt{B^{2} S^{2}+3 \kappa C_{0}^{2}\left[-\Lambda^{2} S^{n}+\left(\sqrt{1+\sigma \varphi_{0}^{2} S^{2} / C_{0}^{2}}-1\right) / \sigma\right]}}=x .
$$


In this case $S(x)$ is finite and its maximum value is defined from

$$
S^{n}(x)=\frac{1}{3 \kappa C_{0}^{2} \Lambda^{2}}\left[B^{2} S^{2}+3 \kappa C_{0}^{2}\left(\sqrt{1+\sigma \varphi_{0}^{2} S^{2} / C_{0}^{2}}-1\right) / \sigma\right] .
$$

Noticing that at spatial infinity effects of nonlinearity vanish, from (3.91) we find

$$
\left.S(x)\right|_{x \rightarrow-\infty} \sim e^{H x} \rightarrow 0,\left.\quad S(x)\right|_{x \rightarrow+\infty} \sim e^{-H x} \rightarrow 0,
$$

with $H=\sqrt{B^{2}+3 \kappa \varphi_{0}^{2} / 2}=B \sqrt{1+\bar{\kappa} / 2}$. In this case the energy density $T_{0}^{0}$ defined by (3.90) is localized and the total energy of the system in bound. Nevertheless, spin and charge of the system unlimited.

Let us go back to the general case. For $F=F(S)$ we now have

$$
T_{1}^{1}=m S-F(S)+2 \Upsilon \frac{d \Psi}{d \Upsilon}-\Psi
$$

It follows that for the arbitrary choice of $\Psi(\Upsilon)$, obeying (2.5), we can always choose nonlinear spinor term that will eliminate the scalar field contribution in $T_{1}^{1}$, i.e., by virtue of total freedom we have here to choose $F(S)$, we can write

$$
F(S)=F_{1}(S)+F_{2}(S), \quad F_{2}(S)=2 \Upsilon \frac{d \Psi}{d \Upsilon}-\Psi,
$$

since $\Upsilon=\Upsilon\left(S^{2}\right)$. To prove this we go back to (2.22) that gives

$$
\Upsilon\left(\frac{d \Psi}{d \Upsilon}\right)^{2}=-\frac{\varphi_{0}^{2} S^{2}}{C_{0}^{2}} .
$$

Since $\Psi$ is the function of $\Upsilon$ only, (3.96) comprises an algebraic equation for defining $\Upsilon$ as a function of $S^{2}$. For (3.95) takes place, we find

$$
\left(\alpha^{\prime}\right)^{2}-B^{2}=-\frac{3 \kappa C_{0}^{2}}{S^{2}}\left[m S-F_{1}(S)\right] .
$$

As we see, the scalar field has no effect on space-time, but it contributes to energy density and total energy of the system as in this case

$$
T_{0}^{0}=S F_{1}^{\prime}(S)-F_{1}(S)+S \frac{d}{d \Upsilon}\left(-2 \Upsilon \frac{d \Psi}{d \Upsilon}+\Psi\right) \frac{d \Upsilon}{d S}+2 \Upsilon \frac{d \Psi}{d \Upsilon}-\Psi .
$$

Note that in (3.94) with $F(S)$ arbitrary, we cannot choose $\Psi(\Upsilon)$ such that

$$
2 \Upsilon \frac{d \Psi}{d \Upsilon}-\Psi=F(S)
$$

due to the fact that $\Psi(\Upsilon)$ is not totally arbitrary, since it has to obey

$$
\lim _{\Upsilon \rightarrow 0} \Psi(\Upsilon) \rightarrow \frac{1}{2} \Upsilon, \quad \lim _{\Upsilon \rightarrow 0} 2 \Upsilon \frac{d \Psi}{d \Upsilon}-\Psi=\frac{1}{2} \Upsilon=\frac{\varphi_{0}^{2}}{2 C_{0}^{2}} S^{2},
$$


whereas at $S \rightarrow 0, F(S)$ behaves arbitrarily.

\subsection{Numerical Solutions}

Let us now numerically solve Equation (2.37). To begin with we rewrite Equation (2.37) for the case with $F=\lambda S^{n}$ :

$$
\alpha^{\prime}=\sqrt{B^{2}-3 \kappa e^{2 \alpha}\left[m S-\lambda S^{n}-a \varphi_{0}^{2} S^{2} / C_{0}^{2}+b\left(1-\sqrt{1+\sigma \varphi_{0}^{2} S^{2} / C_{0}^{2}}\right) / \sigma\right]} .
$$

Recall that here, $\kappa$ is Einstein's gravitational constant, $B$ is some arbitrary constant connecting $\alpha$ with $\beta$ and $\chi, m$ is the spinor mass, $\lambda$ is the spinor field self-coupling constant, $n$ is the power of spinor field nonlinearity, $C_{0}$ is some arbitrary constant connecting $\alpha$ with $S, \sigma$ is the parameter of scalar field nonlinearity and $\varphi_{0}$ is an integration constant that arises while integrating the scalar field equation. The coefficients $a$ and $b$ are to be set as follows: (i) $a=0.5$ and $b=0.0$ corresponds to the linear scalar field; (ii) $a=0.0$ and $b=1.0$ corresponds to the Born-Infeld type nonlinear scalar field. Keeping into mind that $S=C_{0} e^{-\alpha}$ Equation (3.101) can be written in terms of $S$ :

$$
\begin{aligned}
S^{\prime} & =\sqrt{B^{2} S^{2}-3 \kappa C_{0}^{2}\left[m S-\lambda S^{n}-a \varphi_{0}^{2} S^{2} / C_{0}^{2}+b\left(1-\sqrt{1+\sigma \varphi_{0}^{2} S^{2} / C_{0}^{2}}\right) / \sigma\right]} \\
& \equiv \mathscr{F}(S, p),
\end{aligned}
$$

where $p$ is the problem parameters: $p \equiv\left\{B, \kappa, C_{0}, m, \lambda, n, a, b, \varphi_{0}, \sigma\right\}$. As one sees, Equations (3.101) and (3.102) are multiparametric problems. Given the concrete values of the parameters $p$ and the value of $S(\alpha)$ at the starting point $(-\infty)$ one observes the development of $S(\alpha)$ with respect to $x$, hence $\beta, \chi, \varphi, \psi$ and other quantities.

The positivity of the radical in (3.102) imposes some restrictions on $S(\alpha)$ which can be used to choose the value of $S(\alpha)$ at the starting point.

Equations (3.101) and (3.102) are solved numerically for different choice of the problem parameter $p$. Note that the richness of the choice of $p$ gives rise to a large number of interesting results. Only a few of them are illustrated in the foregoing subsections.

\section{CONCLUSION}

The system of nonlinear spinor and nonlinear scalar fields with minimal coupling has been thoroughly studied within the scope of general relativity given by a plane-symmetric space-time. The spinor field nonlinearity $F$ is chosen to be a power law of the invariants $I$ or $J$, whereas the nonlinear scalar field is 
given by a Born-Infeld type Lagrangian. It has been shown that the spinor field is more sensitive to gravitational field than the scalar field. To verify the role of the material field nonlinearity the system of linear spinor and scalar fields are studied as well. As it was expected, that the energy density and the total energy of the linear spinor and scalar field system are not bounded and the system does not possess real physical infinity, hence the configuration is not observable for an infinitely remote observer, since in this case

$$
R=\int_{-\infty}^{\infty} \sqrt{g_{11}} d x=\int_{-\infty}^{\infty} e^{\alpha} d x=\frac{4 C_{0} H}{M^{2}}<\infty
$$

It has been shown that the introduction of nonlinear spinor term into the system eliminates these shortcomings and we have the configuration with finite energy density and limited total energy. In this case the system possesses real physical infinity making the configurations observable. Thus we see, spinor field nonlinearity is crucial for the regular solutions with localized energy density. We also conclude that the properties of nonlinear spinor and scalar field system with minimal coupling are defined by that part of gravitational field which is generated by nonlinear spinor field. It is also shown that together with the spinor field nonlinearity, the gravitational field too plays an important role in the formation of the field configurations with limited total energy, spin, and charge.

\section{REFERENCES}

Adomou, A. and Shikin, G. N. (1998a). Izvestia VUZov, Fizika 41(7), 69.

Adomou, A. and Shikin, G. N. (1998b). Gravitation \& Cosmology 4(2/14), 107.

Alvarado, R., Rybakov, Y. P., Saha, B., and Shikin, G. N. (1995a). JINR Preprint E2-95-16, p. 11.

Alvarado, R., Rybakov, Y. P., Saha, B., and Shikin, G. N. (1995b). Communications in Theoretical Physics 4(2), 247, gr-qc/9603035.

Alvarado, R., Rybakov, Y. P., Saha, B., and Shikin, G. N. (1995c). Izvestia VUZob, Fizika 38(7) 53.

Anguige, K. (2000). Classical and Quantum Gravity 17, 2117.

Berestetski, V. B., Lifshitz, E. M., and Pitaevski, L. P. (1989). Quantum Electrodynamics, Nauka, Moscow.

Bogoliubov, N. N. and Shirkov, D. V. (1976). Introduction to the Theory of Quantized Fields, Nauka, Moscow.

Brill, D. and Wheeler, J. (1957). Review of Modern Physics 29, 465.

Bronnikov, K. A. and Shikin, G. N. (2001). Cylindrically symmetric solitons with nonlinear selfgravitating scalar fields. e-print: gr-qc/0101086.

Bronnikov, K. A. and Shikin, G. N. (1991). Self-gravitating particle models with classical fields and their stability. In Itogi Nauki and Tekhniki (Results of Science and Technology): vol. 2. Gravitation and Cosmology, VINITI, Moscow, pp. 4-55 (in Russian).

Chervon, S. V. and Shabalkin, D. Y. (2000). Gravitation \& Cosmology 21, 41.

da Silva, M. F. A. and Wang, A. (1998). Physics Letters A 244, 462.

Gross, D. J. and Neveu, A. (1974). Physical Review D 10, 3235.

Hehl, F. W., von der Heyde, P., and Kerlick, G. D. (1976). Review of Modern Physics 43, 393. 
Heisenberg, W. (1953). Physica 19, 897.

Heisenberg, W. (1957). Review of Modern Physics 29, 269.

Heisenberg, W. (1968). An Introduction to Unified Field Theory of Elementary Particles, Mir, Moscow. Ivanenko, D. (1938). Phys. Z. Sowjetunion 13, 141.

Ivanenko, D. (1947). Uspekhi Fiz. Nauk 32, 149.

Ivanenko, D. D. (1959). Nonlinear Quantum Field Theory, IL, Moscow, p. 5.

Kibble, T. W. B. (1960). Journal of Mathematical Physics 2, 212.

Mitskievich, N. V. (1969). Physical fields in General Relativity, Nauka, Moscow.

Nouri-Zonoz, M. and Tavanfar, A. R. (2001). Classical and Quantum Gravity 18, 4293.

Ori, A. (1998). Physical Review D 57, 4745.

Pradhan, A. and Pandey, H. R. (2003). International Journal of Modern Physics D 12, 941.

Rabounski, D. D. and Borisova, L. B. (2001). Particles here and beyond the mirror. Editorial URRS, Moscow; e-print gr-qc/0304018.

Ranada, A. F. (1983). Quantum Theory, Groups, Fields and Particles, A. O. Barut, ed., Reidel, p. 271.

Ranada, A. F. and Soler, M. (1972). Journal of Mathematical Physics 13, 671.

Rendall, A. D. (1995). General Relativity and Gravitation 27, 213.

Rodichev, V. (1961). J. Eks. Teor. Fiz. 40, 1469.

Rybakov, Y. P., Saha, B., and Shikin, G. N. (1992). Izvestia VUZov, Fizika 35(10), 112.

Rybakov, Y. P., Saha, B., and Shikin, G. N. (1994a). PFU Reports. Physics 2(2), 61.

Rybakov, Y. P., Saha, B., and Shikin, G. N. (1994b). Communications in Theoretical Physics 3(1), 67.

Rybakov, Y. P., Saha, B., and Shikin, G. N. (1994c). Communications in Theoretical Physics 3, 199.

Rybakov, Y. P., Saha, B., and Shikin, G. N. (1997). International Journal of Theoretical Physics 36(6), 1475.

Rybakov, Y. P., Saha, B., and Shikin, G. N. (1998). Gravitation \& Cosmology 4(2/14), 114.

Saha, B. (2000). International Journal of Modern Physics A 15(10), http://www.jinr.ru/ bijan/my_papers/ijmpa00_1481.pdf.

Saha, B. (2001). Physical Review D 64, http://www.jinr.ru/ bijan/my_papers/PRD23501.pdf.

Saha, B. (2005). Interacting scalar and spinor fields in Bianchi type I universe filled with magneto-fluid. Journal of Astrophysics and Space Science 299(1), 149.

Saha, B. (2004). Physical Review D 69, http://www.jinr.ru/ bijan/my_papers/PRD24006.pdf; e-print: gr-qc/0308088.

Saha, B. and Boyadjiev, T. (2004). Physical Review D 69, http://www.jinr.ru/ bijan/my_papers/ PRD24010.pdf; e-print: gr-qc/0311045.

Saha, B. and Shikin, G. N. (1997a). Journal of Mathematical Physics 38, http://www.jinr.ru/ bijan/my _papers/JMP05305.pdf.

Saha, B. and Shikin, G. N. (1997b). General Relativity and Gravitation 29, http://www.jinr.ru/ bijan/my _papers/grg97_1099.pdf.

Saha, B. and Shikin, G. N. (2004). Chezkoslovak Journal of Physics 54(6), http://www.jinr.ru/ bijan/my _papers/czjp54_597.pdf.

Schweber, S. S. (1961). An Introduction to Relativistic Quantum Field Theory, Harper \& Row, New York, NY.

Sciama, D. W. (1960). Festschrift for Infeld, Pergamon Press, p. 415.

Shikin, G. N. (1991). Academy of Science USSR 19, 21. Preprint IPBRAE.

Shikin, G. N. (1995). Basics of Soliton Theory in General Relativity, URSS Publishers, Moscow.

Taruya, A. and Nambu, Y. (1996). Progress in Theoretical Physics 95, 295.

Taub, A. H. (1951). Annals of Mathematics 53, 472.

Taub, A. H. (1956). Physical Review 103, 454.

Thirring, J. K. and Skyrme, T. H. R. (1962). Nuclear Physics 31, 550.

Utiyama, R. (1956). Physical Review 101, 1596. 
Weyl, H. (1950). Physical Review 77, 699.

Yazadjiev, S. S. (2003). Classical and Quantum Gravity 20, 3365.

Zelmanov, A. L. and Agakov, V. G. (1989). Elements of General Relativity, Nauka, Moscow.

Zhelnorovich, V. A. (1982). Spinor Theory and Its Application in Physics and Mechanics, Nauka, Moscow.

Zhuravlev, V. M., Chervon, S. V., and Shabalkin, D. Y. (1997). Gravitation \& Cosmology 4, 312. 